\title{
Damaris Nübling
}

\section{Zwischen Konservierung, Eliminierung und Funktionalisierung: der Umlaut in den germanischen Sprachen}

\section{Einleitung}

Alle germanischen Sprachen haben in den nachchristlichen Jahrhunderten eine phonologische Umlautphase durchlaufen, allerdings mit je unterschiedlichen Resultaten. Dieser Umgang mit den Umlautprodukten wurde bisher nie vergleichend in den Blick genommen; vielmehr bekommt man in jeder Einzelphilologie den Eindruck, als habe die Umlautentwicklung nur so und nicht anders verlaufen können. Erst die historischkontrastive Perspektive erweist, dass sich drei Pfade systematisieren lassen: Der Umlaut wird konserviert (Isländisch), er wird eliminiert (Englisch, Niederländisch) - Schwedisch nimmt hier eine Zwischenposition ein -, oder er wird funktionalisiert (grammatikalisiert) und damit morphologisch ausgedehnt (Deutsch, Luxemburgisch).

Im Folgenden werden diese drei Wege nicht nur beschrieben, sondern auch begründet. Der konsequente Sprachwandelvergleich ermöglicht dabei das Verständnis von Zusammenhängen und erlaubt es, aus den Einzelphilologien abgeleitete Annahmen zu revidieren.

\section{Die phonologische Umlautphase}

Mit „Umlaut“ ist hier der $i$-Umlaut ( $i$-UL), eine fernassimilatorische Palatalisierung (Frontierung), gemeint. Die erste Umlautphase, die in der Hebung von $e>i$ (vor $i, j, u$ ) und Senkung von $i>e$ und $u>o$ (vor $a, e, o$, auch $a$-Umlaut genannt) besteht, bleibt hier ausgeklammert, ist aber auch im Zusammenhang verschiedener Umlautphänomene zu sehen. ${ }^{1}$

In diesem Beitrag soll weniger der phonetisch/phonologische Aspekt der Umlautung als seine Weiterentwicklung (nach der Umlautphase) in den germanischen Sprachen im Vordergrund stehen. Allerdings muss knapp auf die phonologische Umlautphase eingegangen werden, spielt doch z.B. die Dauer dieser Umlautphase bzw., genauer, der Erhalt des umlautauslösenden Palatals (meist $-i(-)$ bzw. $-j-$ ), eine wichtige Rolle für die Wahrscheinlichkeit seiner Phonologisierung (Ronneberger-Sibold 1990) ${ }^{2}$ bzw. sogar (schon in der gleichen Phase) der Morphologisierung des Umlautprodukts (Dal 1971).

1 Hebung und Senkung beziehen sich auf die Stellung bzw. Höhe der Hinterzunge. Der $a$-Umlaut wird manchmal auch „Brechung“ genannt, was hier vermieden wird.

2 Präziser ist der Terminus „Umlautphonemisierung“, wie ihn z.B. Schulte (1998) verwendet. 
Man ist sich einig darüber, dass der $i$-Umlaut eine Nord-Süd-Bewegung vollzogen hat, d.h. im Norden der Germania früher und regelhafter durchgeführt wurde als im Süden. Was das alemannisch-schweizerdeutsche Sprachgebiet betrifft, so spricht man hier sogar von der oberdeutschen Umlautfeindlichkeit. Dennoch - und dieses Paradox ist hier auch zu behandeln - ist es ausgerechnet das oberdeutsche Gebiet, das die Umlaut-Funktionalisierung am weitesten vorangetrieben hat.

Der $i$-Umlaut besteht in einer Palatalisierung von Velarvokalen und -diphthongen, d.h. die vordere Zungenstellung wird regressiv auf die Velarvokale übertragen. Dabei behalten /o/ und / $\mathrm{u}$ / (sowie deren Längen und Diphthonge) ihre Lippenrundung: Hier entstehen also qualitativ neue Laute, die erstmals Lippenrundung mit palataler Zungenstellung kombinieren (Mottausch 2002 spricht hier treffend von „Mischvokalen“). Im Fall der $a>e$ Umlautung ist dies anders: Hier erfolgt eine Hebung der Zunge, und das Umlautprodukt ist in der Regel, zumindest nach einiger Zeit, mit dem alten (germ.) $e$ zusammengefallen, vgl. nhd. $h[\varepsilon]$ lfen $(<$ germ. $e$ ) und $r[\varepsilon]$ nnen $(i$-Umlaut $<$ germ $a)$.

In manchen Sprachen (z.B. Englisch) wurden die gerundeten Umlautvokale entrundet (Kastovsky 1994). Diese fielen dann mit $i$ - bzw. $e$-Phonemen zusammen. Hier hat also keine Phonologisierung (= Phonemisierung) und damit auch keine Phonemspaltung stattgefunden. Das andere Extrem findet sich im Deutschen, wo der Umlaut zwischenzeitlich (im Mittelhochdeutschen) zu neun neuen Phonemen geführt hat, die später, v.a. nach dem Zusammenfall der verschiedenen $e$-Laute, verringert wurden. Bis in die 1970er Jahre hinein glaubte man, dass sich die Phonemisierung der Umlautprodukte in dem Moment vollzog, als die palatalen Auslöservokale zu Schwa reduziert wurden bzw. (später) schwanden. Ab da wurden die Umlautprodukte auch verschriftlicht. Dies führte zu einer zu späten Datierung v.a. des sog. Sekundärumlauts ins Mittelhochdeutsche. Heute weiß man, dass Primär- und Sekundärumlaut zur gleichen Zeit stattfanden. Das Umlautprodukt von kurzem $e<a$ wurde deshalb schon im Althochdeutschen verschriftet, weil es dicht an dem ohnehin schon vorhandenen ererbten /e/-Phonem < germ. $e$ (in Handbüchern oft mit <ë> bezeichnet) lag, wobei seine Aussprache [e] geschlossener war als die von germ. $\ddot{e}[\varepsilon]$ (Braune \& Reiffenstein 2004: 31). ${ }^{3}$ So lag es nahe, das dafür schon bestehende Graphem für diesen ,neuen“ Laut mitzuverwenden, auch wenn der Umlautauslöser noch vorhanden war (<gesti>). Allerdings gab es in Handschriften auch graphematische Differenzierungen zwischen den beiden Lauten. Im Fall der anderen Umlautprodukte war das anders, hier mussten neue Grapheme bzw. Diakritika gefunden werden. Solange der $i$-Laut in der Folgesilbe noch bestand, erübrigte sich eine eigene Verschriftung, da die Qualität des Umlauts aus dem Kontext erschließbar war. ${ }^{4}$

Die Phonemisierung der Umlaute hat also vor der Endsilbenabschwächung stattgefunden: \#...[UL] ...i/j\# > \#.../UL/ ...i/j\# (die genaue Domäne ist dabei der phonologische

3 Die beiden $e$-Laute (germ. $\ddot{e}$ und der Umlaut von $a$ ) waren noch im Mittelhochdeutschen getrennt und reimten nicht aufeinander. Manche Dialekte haben bis heute diese beiden Phoneme erhalten. Im Standard sind sie indessen zusammengefallen. - An dieser Stelle sei einem anonymen Gutachter für wertvolle Präzisierungen und Ergänzungen gedankt.

4 Dies bedeutet jedoch nicht im Umkehrschluss, dass Nichtverschriftung des Umlautprodukts auf fehlende Phonemisierung hinweist: Besonders die Umlaute von $o$ und $u$ bleiben vielerorts lange unbezeichnet. Ebenso müssen zeitlich versetzte Umlautbezeichnungen nicht auf zeitlich versetzte Phonemisierungen hindeuten. 
Fuß). Wann dies geschah, lässt sich nicht exakt rekonstruieren, doch hat die lange Phase des $i$-Erhalts im Althochdeutschen die Phonemisierung, d.h. die Autonomisierung der Umlaute gefördert: Ronneberger-Sibold (1990) geht plausiblerweise von einem längeren Stadium aus, in dem das palatale Folgesegment mehr oder weniger deutlich, abhängig auch von Lento vs. Allegro, ausgesprochen wurde, so wie dies für heute ablaufende Reduktionsprozesse auch gilt. Dabei muss eine Gewichtsverlagerung stattgefunden haben, d.h. eine (regressiv wirkende) Bedingung (i-Segment: also Umlautung des Velars) wurde nach und nach (auch dies verläuft nicht sprunghaft) zu einer (progressiv wirkenden) Indizierung: Das [y] (stellvertretend für alle Umlautvokale) wurde zum Index für folgendes $i$ (oder $j$ ) und hat mit dem damit verbundenen Zugewinn an „Signalstärke“ (Kazzazi 2004: 158) die neue Funktion erlangt, den folgenden Palatal anzukündigen (es enthält ihn ja schon materiell):

Der eigentlich bedeutsame Vorgang bei der Phonologisierung scheint uns in der „Indexikalisierung der neuen Einheit", in diesem Fall der Umlaut-Vokale, zu bestehen. [...] Die logische Relation des Bedingens, auf der die strukturelle des Dominierens beruht, verläuft weiterhin von alter [ $=i$-Laut D.N.] zu neuer Einheit [= Umlaut - D.N.], die semiotische des Indizierens jedoch von der neuen zur alten. [...] In ihrer kommunikativ relevanten Rolle als Index hat also die neue Einheit die für ihr Überdauern unerlässliche Eigenständigkeit noch vor dem Schwund der alten erreicht - die logische und die strukturelle Eigenständigkeit folgt nahezu automatisch nach. (RonnebergerSibold 1990: 194).

D.h. die Autonomisierung der Umlautprodukte hat bereits in einem Stadium stattgefunden, als $i$ bzw. $j$ noch vorhanden waren, aber gar nicht mehr den Umlaut phonetisch auslösten. Nur aufgrund der Tatsache, dass es nach und nach die Umlautvokale sind, die nun ihrerseits den Folgelaut anzeigen, lässt sich deren Fortbestehen nach der Nebensilbenabschwächung erklären. Die Phonemisierung im Stadium der vollen Nebensilben illustriert RonnebergerSibold an folgendem Beispiel: vorahd. [suntia] $>$ ahd. [syntja] $>$ [synta] (9. Jh.) 'Sünde' Indem hier der Umlaut [y], der sich ja durch das gesamte Paradigma zieht (vgl. nhd. Sünde), keine morphologische Funktion versieht (im Gegensatz zu den oft herangezogenen Pluralbeispielen), stellt sich Ronneberger-Sibold gegen die These von Dal (1971), die noch weiter geht und schon in dieser frühen Phase eine Umlautmorphologisierung ansetzt: $\# \ldots\{$ UL $\}$...i/j\#. Diese wiederum macht sie für die Phonemisierung der Umlautvokale verantwortlich:

Prinzipiell kann man sagen, dass Differenzierung der Laute [Umlaute - D.N.] dann aufrechterhalten wurde, wenn sie als konstitutives Element bei der Unterscheidung von morphologischen Kategorien empfunden wurde. Wenn die Umlautsqualität des Pluralvokals in mhd. züge nach Schwinden der $i$-Qualität der Endung aufrechterhalten bleibt, so ist der Grund der, dass der Umlautswechsel ein zusätzliches Pluralmorphem geworden ist. Hier stehen wir meines Erachtens an dem entscheidenden Punkt für das Verständnis der deutschen Umlautsvorgänge: man muss, um sie zu begreifen, aus der phonologischen Ebene heraustreten und morphologische Gesichtspunkte heranziehen. Und zwar nicht nur sekundär zur Erklärung des „analogischen“ Umlauts, sondern ganz primär bei der Konstituierung der Umlautphoneme. (Dal 1971: 36)

Auch wenn diese Argumentation zu strikt ist (Dal diskutiert ausschließlich Beispiele, in denen der Umlaut eine morphologische Funktion ausübt), so bestreitet auch RonnebergerSibold nicht, dass der Umlaut - unabhängig von seiner Phonologisierung - schon sehr früh 
für funktionale Zwecke eingesetzt wurde. Dies soll jedoch später behandelt werden (in Abschn. 2).

Für das Skandinavische liegt mit Schulte (1998) eine sehr detaillierte Untersuchung zum phonetischen und phonemischen $i$-Umlaut vor. Die Phonemisierung der Produkte datiert er ins 6. Jh. Ähnlich wie im Deutschen wurden sämtliche Velarvokale umgelautet. Für das Alt- und das Neuisländische zeigt dies Tabelle 1.

Tabelle 1: Die Palatalisierung im Späturnordischen und die Fortsetzung der Umlaute im Alt- und Neuisländischen ${ }^{5}$

\begin{tabular}{|c|c|c|c|c|c|}
\hline \multirow{2}{*}{$\begin{array}{l}\text { vor urn. } \\
\bar{l}, \bar{l}, j \text { etc. }\end{array}$} & \multicolumn{2}{|c|}{$>$ altnordisch } & \multicolumn{2}{|c|}{ > neuisländ. } & neuisländische Beispiele \\
\hline & / I & $<>$ & 11 & $<>$ & \\
\hline$a$ & $/ \mathfrak{e}>\mathrm{e} /$ & $\langle e\rangle$ & $/ \varepsilon(:) /$ & $\langle e\rangle$ & fer 'fährt' (zu fara 'fahren') \\
\hline $\bar{a}$ & /æ:/ & $\langle æ\rangle$ & /ai(:)/ & $\langle æ\rangle$ & gratur 'weint' (zu gráta 'weinen') \\
\hline$o$ & $/ œ /$ & $\langle\emptyset\rangle$ & $/ œ(:) /$ & $\langle\ddot{0}\rangle$ & selten ( $\ddot{o}$ meist aus $u$-umgelautetem $a$ ) \\
\hline $\bar{o}$ & /œ:/ & 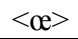 & /ai(:)/ & $\langle æ>$ & staerri 'größer' (zu stór 'groß’) \\
\hline$u$ & /y/ & $\langle y\rangle$ & /i(:)/ & $\langle y\rangle$ & yngri 'jünger' (zu ungur 'jung') \\
\hline $\bar{u}$ & /y:/ & $\langle\dot{y}\rangle$ & /i(:)/ & $\langle y ́>$ & sýpur 'säuft' (zu súpa 'saufen') \\
\hline$a u$ & /عy/ & $\langle e y\rangle$ & $/ \varepsilon \mathrm{i}(:) /$ & $\langle e y\rangle$ & hleypur 'läuft' (zu hlaupa 'laufen') \\
\hline
\end{tabular}

Die phonetischen Umlautprozesse waren jedoch komplizierter als im Deutschen. Hinzu kommt, dass die in der 2. Spalte von Tabelle 1 enthaltenen Umlautprodukte teilweise auch über andere Umlautprozesse entstehen konnten, was in der linken Spalte ganz oben durch „etc.“ angedeutet wird: So löste auch das palatale $-R(<$ germ. $-z<$ idg. $-s)$ eine Palatalisierung aus, allein zwar nur von direkt vorangehendem Vokal (vgl. kýr 'Kuh' < urn. $* k \bar{u} R$ ), in Kombination mit (ansonsten Kurzstämme nicht umlautendem) $i$ auch generell (s. Schulte 1998: 244-248). Hier wirken also kombinatorisch zwei Palatallaute zusammen. Dies gilt auch (wenngleich im Detail anders) für den sog. verstärkten $i$-Umlaut (auch $g i / k i$ Umlaut genannt): Wenn im Urnordischen (Ende des 6. Jhs. nach der eigentlichen Umlautphase) ein sekundäres $i$ (das normalerweise keinen Umlaut auszulösen vermochte) direkt auf ein $g$ oder $k$ folgte, so nahmen diese Konsonanten die Palatalität an und verstärkten sie so, dass vorausgehende Velarvokale umgelautet wurden (vgl. nisl. degi 'Tag (Dat. Sg.)' $<$ urn. *dagi $<$ dagē $)$.

Während es sich bis jetzt um verstärkte und erweiterte Palatalisierungen handelt, konnten im Altnordischen scheinbare $i$-Umlautprodukte auch auf die Labialisierung von Palatalen zurückgehen, d.h. ein und derselbe Laut konnte auf unterschiedlichen Umlauten unterschiedlicher Basisvokale beruhen (s. Abb. 1). Dies könnte als ein Grund für ihre Nichtfunktionalisierung angesehen werden.

5 Grundsätzlich hängt die Quantität der Vokale im heutigen Isländischen von der betreffenden Silbenstruktur ab (s. die eingeklammerten Längezeichen). Im weiteren Verlauf wird auf diese Einklammerungen verzichtet. 


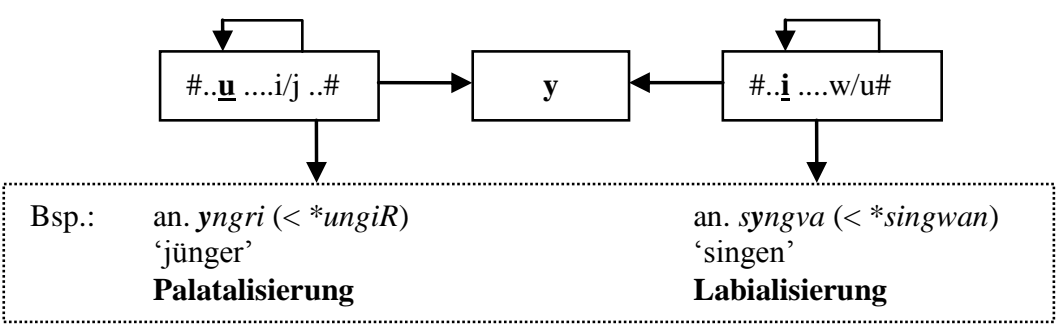

Abbildung 1: Zwei Wege zu [y]: Palatal- und Labialumlaut im Späturnordischen

Ebenso konnten auch $\bar{l}>\bar{y}$ sowie verschiedene (lange und kurze) $e$-Laute zu $\phi$-Lauten labialisiert werden (was Abb. 1 nicht zeigt; hierzu s. Haugen 1982: 31). Die meisten heutigen $/ \varnothing /$-Vokale <ö> gehen indessen auf urn. kurzes $a$ zurück, das vor $w$ - bzw. $u$ haltiger Silbe labialisiert wurde (sog. $u$-Umlaut) und zunächst (noch im Altnordischen) ein eigenes Phonem bildete, nämlich $/ 0 /$, geschrieben $<_{0}>$. Dieses offene, velare $/ 0 /$ ist später mit gerundetem $/ \varnothing /(\mathrm{zu} / \varnothing /)$ zusammengefallen und auch entsprechend verschriftet worden: urn. *barn-u 'Kind (Nom./Akk. Pl.)' > an. born > isl. börn, dän. børn 'Kinder'.

Wie Tabelle 1 zeigt, hat sich die Aussprache mancher Umlautprodukte im Neuisländischen stark verändert; auch sind Phonemzusammenfälle zu konstatieren, z.B. von an. /æ:/ und /œ:/ zu nisl. /ai/ <æ> sowie von an. /y/ und /i/ zu nisl. /i/ (auch von deren Längen).

Damit wird deutlich: Im Gegensatz zu den westgermanischen Sprachen können palatale Labialvokale im Nordgermanischen mindestens zwei Entstehungswege (und damit Basisvokale) haben (s. auch Dal 1971: 44). Dagegen besteht in den westgermanischen Sprachen eine weitgehende 1:1-Beziehung bzw. -Ableitbarkeit zwischen Umlaut und Basisvokal zumindest bei den gerundeten Umlautvokalen (fast jedes $\ddot{o}$ geht auf ein $o$ zurück, fast jedes $\ddot{u}$ auf ein $u$ etc. - abgesehen von spontanen, lexikalisierten Rundungen wie helle > Hölle, finf > fünf). Ob bzw. wie gegebenenfalls diese zahlreichen Umlaute funktionalisiert wurden, ist Thema von Abschnitt 2.3.

Für das Englische gilt, dass es schon im (dialektal sehr stark differenzierten) Altenglischen zu gerundeten Umlautphonemen kam, nach Faiß (1989: 27) bereits im 6. Jh. Die Endsilbenabschwächung trat bedeutend früher ein als im Deutschen (Dal 1971, Penzl 1994: 27-28). Diese Umlaute wurden jedoch schon wenig später - teilweise noch in der altenglischen Phase, genauer zwischen dem 9. und 11. Jh. - entrundet. Mottausch (2002: 89) sieht den Grund darin, dass ,in keiner altenglischen Mundart das Prinzip Umlaut = Grundvokal mit hoher (=i) Zungenstellung ausnahmslos gewahrt ist", sondern durch weitere lautliche Entwicklungen ,überall mehr oder weniger durchlöchert“ ist. D.h. durch bspw. die Verdumpfung (Rundung) von ae. $a>q$ entstand die Asymmetrie gerundeter Basisvokal - ungerundeter Umlaut (also $q-\ddot{a}$, später $>e$ ). Damit sei das „Prinzip der eindeutigen Entsprechungen“ (13) durchbrochen gewesen, was die Eigenentwicklungen der Umlaute (Entrundung) begünstigt habe.

Mit Blick auf viele andere germanische Sprachen, v.a. das Luxemburgische, aber auch das Schwedische und das Isländische (s. Tab. 1), wo solche asymmetrischen Zuordnungen (nichteindeutige Entsprechungen) erhalten blieben, erscheint diese Begründung, die ähnlich auch Kastovsky (1994) vertritt, nicht allzu plausibel. Schwerer als eine sich nur durch [ \pm palatal] auszeichnende 1:1-Alternanz zwischen Basis- und Umlautvokal scheint m.E. die 
Tatsache zu wiegen, ob eine morphologische Distribuierung (oder gar Funktionalisierung) dieser Alternanzen gegeben ist. Bei solchen Überlegungen wird der Nutzen sprachvergleichender Untersuchungen offenkundig.

Im Schwedischen, das hier stellvertretend für das Festlandskandinavische stehen soll (also neben Schwedisch Dänisch, Nynorsk und Bokmål), wurde langes an. $a$ [a:] zu å [o(:)] verdumpft, ohne dass die strukturelle Relation Basisvokal - Umlaut aufgegeben worden wäre: stång - stänger 'Stange - Stangen', små - smärre 'klein (Pl.) - kleiner'. Auch wurde langes an. $o ́$ [o:] mehrheitlich zu [u:] verschoben, das heute noch mit [œ(:)] alterniert, vgl. fot [fu:t] - fötter [foet:er] 'Fuß - Füße', stor [stu:r] - större [stor:e] 'groß - größer'. Nach Holmes \& Hinchliffe (1994: 18, 108-109) alternieren außerdem noch: $a-\ddot{a}$ (and - änder 'Ente - Enten'), $o-\ddot{o}$ (bonde - bönder 'Bauer - Bauern') sowie $u-y$ (ung - yngre 'jung jünger').

Im Niederländischen ist die phonologische Umlautbasis dagegen schon immer geringer ausgeprägt gewesen als in den anderen germanischen Sprachen. Hier wurden nur die beiden Kurzvokale $a>\ddot{a}>e(=\operatorname{sog}$. Primärumlaut) und $u>y$ (Sekundärumlaut) umgelautet (kurzes $o$ fällt kaum ins Gewicht, da extrem selten vor $i$ vorhanden). Langvokale und Diphthonge wurden nicht umgelautet bzw., präziser, phonemisiert (allophonisch dürften Umlaute vorhanden gewesen sein). Doch verhält es sich in den niederländischen Dialekten ganz anders: Hier stößt ein umlautfreundlicher Osten auf einen umlautfeindlichen Westen (zu den Verhältnissen in den Dialekten s. Goossens 2002a-d, MAND I, II). Im Standard sind beide Umlautprodukte schon früh mit anderen Phonemen zusammengefallen (kurzes $u$ erfuhr in jeder Position eine spontane Palatalisierung, es wurden also alle $/ \mathrm{u} />/ \mathrm{y} /$ ). D.h. im Niederländischen (wie im Englischen) sind langfristig keine eigenen Phoneme entstanden.

Für das Deutsche, das hier bereits zur Sprache kam, kann man für die althochdeutsche Stufe im Prinzip die Umlaute des Altnordischen in der 2. (grau hinterlegten) Spalte von Tabelle 1 zugrunde legen (es kommt noch $a>\ddot{a}$ [æ] für das vom Primär-Umlaut nicht erfasste, doch später umgelautete kurze $a$ hinzu, sowie $o u>\ddot{o} u$ und $u o>\ddot{u}$ ). Die drei

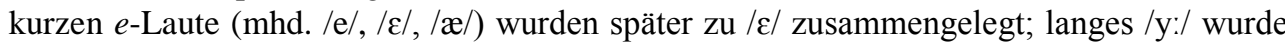
$\mathrm{zu} / \mathrm{i} /<$ äu/eu> diphthongiert und fiel mit dem Diphthongwandelprodukt von mhd. /öu/ zusammen. Ebenso ist das offene Umlaut-/æ:/ mit dem langen /e:/ zusammengefallen (nicht in allen Dialekten, aber im Standard). Mhd. /üa/ wurde zu nhd. /y:/ monophthongiert. Hinzu kommt die frühnhd. Dehnung in offener Tonsilbe, die die Längen neu ordnet. Insgesamt besteht jedoch in der Flexion und oft auch in der Wortbildung heute noch eine Art „Prinzip der eindeutigen Entsprechungen“ (Mottausch 2002: 13) insofern, als weitestgehend (von Fremdwörtern und Eigennamen immer abgesehen) einem $\ddot{u}$ ein $u$ und einem $\ddot{o}$ ein $o$ entspricht (andere Quellen als den Umlaut gibt es kaum). ${ }^{6}$ Darüber hinaus entspricht jedem

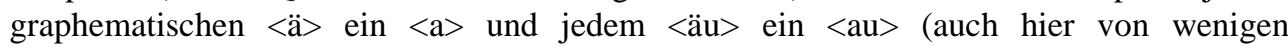
Ausnahmen abgesehen).

Genau dieses Prinzip wurde im Luxemburgischen durch vielfältige phonologische (oft silbenabhängige) Prozesse gründlich durchbrochen: Hier haben sich zum einen die Umlautprodukte, aber auch die Basisvokale z.T. radikal weiterentwickelt, d.h. die 1:1Relation zwischen einstigem Basis- und seinem Umlautvokal ist in beide Richtungen zerstört: Erstens muss der Basisvokal nichts mehr mit Velarität und der Umlautvokal mit

6 Abgesehen von sporadischen Rundungen, s.o. 
Palatalität zu tun haben. Zweitens können einem Basisvokal zwei oder mehr verschiedene ,Umlaut'-Produkte entsprechen (was von der Umgebung und der Silbenstruktur, in die er eingebettet war/ist, abhängt), z.B. entspricht heutigem /uə/ $(<\mathrm{mhd}$. /o/, /a/) sowohl /i:/, /i/ als auch $/ a /<\ddot{e}>$. Drittens können - umgekehrt - dem Umlaut-Produkt mehrere mögliche Basisvokale zukommen: So entspricht heutigem /i/ (< mhd. /y/) sowohl /u/, /uə/ als auch /iə/. Dieses ,Wirrwarr' findet sich in Abbildung 4 in Abschnitt 2.3 dargestellt und hat das Luxemburgische nicht daran gehindert, diese asymmetrischen Zuordnungen zu morphologisieren: Teilweise sind solche Alternanzen sogar heute noch produktiv (Nübling 2001, 2006). Diese Verhältnisse rechtfertigen es, beim luxemburgischen Umlaut von einer Arbitrarisierung bzw. ,Ablautisierung' zu sprechen, denn synchron verhalten sich die auf Umlaut und Ablaut zurückgehenden Reflexe ähnlich.

Der gesamte phonologische Umlautungsprozess kann mit Szczepaniak (2007: 80-83) als eine Schwächung der betonten Vokale angesehen werden, sowie, indem die Zungenposition des Folgepalatals antizipiert wird, als Artikulationserleichterung. Insgesamt sprechen solche Prozesse für typologische Silbensprachlichkeit. Die prosodische Umlautdomäne ist dabei der phonologische Fuß (wie immer dieser sich im Einzelnen definiert, denn dies wird besonders für die historischen Sprachstufen kontrovers diskutiert, und er verändert sich diachron). ${ }^{7}$

\section{Die weiteren Entwicklungen nach der Umlautphase: Konservierung, Eliminierung, Funktionalisierung}

In diesem Abschnitt sollen nun die drei wichtigsten Möglichkeiten, mit den Umlautprodukten umzugehen, skizziert werden. Dies lässt sich mit Abbildung 2 darstellen, bestückt mit den hier zu behandelnden Sprachen. Diese Abbildung zeigt, dass Konservierung den unmarkierten Erhalt der lautgesetzlich entstandenen Verhältnisse darstellt, die im Laufe der Zeit abgebaut (Eliminierung) oder ausgebaut werden können (Funktionalisierung). Dabei besteht Unidirektionalität (s. die Pfeile). Einmal aufgebaute Funktionalisierungen können auch wieder abgebaut werden, was der untere Pfeil zeigt. Dies sind grob gesagt die drei Eckpfeiler, die sich bei genauerem Hinsehen weiter ausdifferenzieren.

7 Einen sog. progressiven $j$-Umlaut haben vom 10.-12. Jh. die ostskandinavischen Sprachen Schwedisch, Dänisch und Norwegisch (auch Nynorsk nach Haugen 1982: 47) vollzogen. Im Unterschied zum regressiven $i$-Umlaut musste $j$ direkt vor dem Velarvokal in der betonten Silbe stehen: an. hjarta 'Herz' > schwed. hjärta ( $h$ - ist stumm), an. mjolk 'Milch' > schwed. mjölk, an. fljúga 'fliegen' > schwed. flyga. Da dieser Umlaut nur auf lexikalischer Ebene wirkt, also ohne grammatische Konsequenzen ist, wird er hier ausgeklammert. 


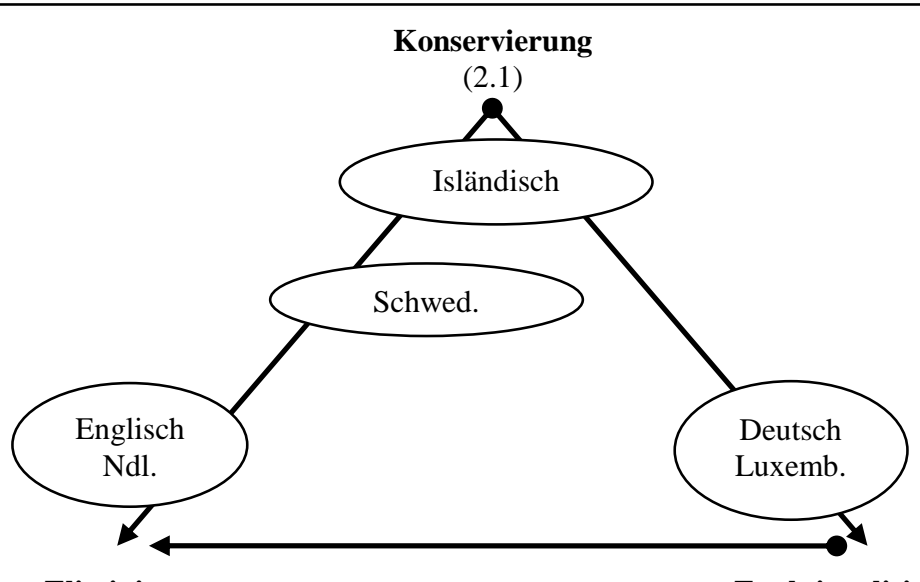

Eliminierung

(2.2)

Funktionalisierung

(2.3)

Abbildung 2: Der Umlaut zwischen Konservierung, Eliminierung und Funktionalisierung

\subsection{Konservierung (Isländisch)}

Die Sprache, die am meisten phonologisch entstandenen Umlaut konserviert hat, ist das Isländische: Hier durchkreuzt der $i$-Umlaut fast wie noch zu späturnordischen Zeiten die Flexionsparadigmen. Weder haben drastische Tilgungen noch Ausdehnungen stattgefunden: Der Umlaut verharrt dort, wo er einst entstanden ist. Kategoriengesteuerte Neuordnungen sind kaum erkennbar. Als einfaches Beispiel sei das Paradigma von isl. dagur 'Tag' aufgeführt (Tab. 2), wo nur der Dat. Sg. verstärkten gi/ki-Umlaut erfahren hat (und der Dat. Pl. regulären $u$-Umlaut).

Tabelle 2: Das Paradigma von isl. dagur 'Tag' (mask. $a$-Klasse)

\begin{tabular}{|c|c|c|c|c|c|}
\hline Num. & Kasus & $\begin{array}{l}\text { urn. Endung } \\
(200-500)\end{array}$ & Alt-/ & Neuisl. & $\begin{array}{l}\text { phon. Prozesse im Späturn. } \\
(500-700)\end{array}$ \\
\hline $\mathrm{Sg}$. & $\begin{array}{l}\mathrm{N} \\
\mathrm{G} \\
\mathrm{D} \\
\mathrm{A}\end{array}$ & $\begin{array}{l}-a R \\
-a s \\
-\bar{e}(>i) \\
-a\end{array}$ & $\begin{array}{l}\text { dag-rl } \\
\text { dag-s/ } \\
\text { deg-i/ } \\
\text { dag- } \emptyset\end{array}$ & $\begin{array}{l}\text { dagur } \\
\text { dags } \\
\text { degi } \\
\text { dag }\end{array}$ & verstärkter gi/ki-UL \\
\hline Pl. & $\begin{array}{l}\mathrm{N} \\
\mathrm{G} \\
\mathrm{D} \\
\mathrm{A}\end{array}$ & $\begin{array}{l}-\bar{o} R \\
-\bar{o} \\
-u m R \\
-a n\end{array}$ & $\begin{array}{l}\text { dag-arl } \\
\text { dag-al } \\
\text { dogum/ } \\
\text { dag-al }\end{array}$ & $\begin{array}{l}\text { dagar } \\
\text { daga } \\
\text { dögum } \\
\text { daga }\end{array}$ & $u$-UL \\
\hline
\end{tabular}

Im Gegensatz zum Westgermanischen trat im Nordgermanischen zusätzlich $u$-Umlaut (Labialisierung) ein (kurzes betontes $/ \mathrm{a} />/ \mathrm{J} /$ vor $u / w$ ). Auch hier haben keine morpho- 
logischen Ausgleiche stattgefunden, s. das Paradigma von isl. völlur 'Feld' in Tabelle 3, für das im Ur- und Altnordischen die Endungen segmentiert werden. Wieder wird deutlich, dass das Neuisländische kaum geneuert hat: Bis auf den Akk. Pl. konserviert es die altnordischen Verhältnisse. Die urn. Wurzel *valp- (etym. zu Wald gehörig) enthielt ein $a$, das im Alt- und Neuisländischen nur noch im Gen. Sg. und Gen. Pl. an der Oberfläche erscheint.

Tabelle 3: Das Paradigma von isl. völlur 'Feld' (mask. $u$-Klasse)

\begin{tabular}{|c|c|c|c|c|c|}
\hline Num. & Kasus & $\begin{array}{l}\text { Wurzel + Endung } \\
\text { im Urnordischen } \\
(200-500)\end{array}$ & $\begin{array}{l}\text { Altnordisch } \\
\text { (ab 8.Jh.)/ }\end{array}$ & Neuisländisch & $\begin{array}{l}\text { phon. Prozesse } \\
\text { im Späturn. } \\
(500-700)\end{array}$ \\
\hline Sg. & $\begin{array}{l}\mathrm{N} \\
\mathrm{G} \\
\mathrm{D} \\
\mathrm{A}\end{array}$ & $\begin{array}{l}\text { valp-uR } \\
\text { valp-o } R>-\boldsymbol{a} R \\
\text { valp-iu } \\
\text { valp-u}\end{array}$ & $\begin{array}{l}\text { voll-rl } \\
\text { vall-arl } \\
\text { vell-il } \\
\text { voll- } \emptyset /\end{array}$ & $\begin{array}{l}\text { völlur } \\
\text { vallar } \\
\text { velli } \\
\text { völl }\end{array}$ & $\begin{array}{l}u \text {-UL } \\
i \text {-UL } \\
u \text {-UL }\end{array}$ \\
\hline Pl. & $\begin{array}{l}\mathrm{N} \\
\mathrm{G} \\
\mathrm{D} \\
\mathrm{A}\end{array}$ & 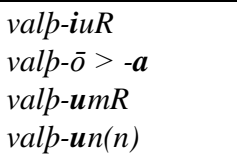 & $\begin{array}{l}\text { vell-ir/ } \\
\text { vall-al } \\
\text { voll-um/ } \\
\text { voll-u/ }\end{array}$ & $\begin{array}{l}\text { vellir } \\
\text { valla } \\
\text { völlum } \\
\text { velli }\end{array}$ & $\begin{array}{l}i \text {-UL } \\
u \text {-UL } \\
u \text {-UL }\end{array}$ \\
\hline
\end{tabular}

Jenseits von $i$ - und $u$-Umlaut gab es weitere Kontaktphänomene, die den Vokalismus affiziert haben. Das Paradigma von isl. fjörður 'Fjord' (Tab. 4) zeigt, wie die erhaltene $e>i$ Hebung und die Brechung ${ }^{8}$ zu tiefgreifenden Stammalternanzen führen können. Heute erscheint die urn. Wurzel *ferp- überhaupt nicht mehr an der Oberfläche.

Tabelle 4: Das Paradigma von isl. fjörður 'Fjord' (mask. $u$-Klasse)

\begin{tabular}{|c|c|c|c|c|c|}
\hline Num. & Kasus & $\begin{array}{l}\text { Wurzel + Endung } \\
\text { im Urnordischen } \\
(200-500)\end{array}$ & $\begin{array}{l}\text { Altnordisch } \\
\text { (ab 8.Jh.)/ }\end{array}$ & Neuisländisch & $\begin{array}{l}\text { phon. Prozesse } \\
\text { im Späturn. } \\
(500-700)\end{array}$ \\
\hline Sg. & $\begin{array}{l}\mathrm{N} \\
\mathrm{G} \\
\mathrm{D} \\
\mathrm{A}\end{array}$ & $\begin{array}{l}*_{f e r p-u R} \\
*_{f e r p-o} R>-a R \\
*_{f e r p-i \underline{u}}>\text { firp-iu } \\
*_{f e r p-u}\end{array}$ & $\begin{array}{l}\text { fjord-rl } \\
\text { fjard-arl } \\
\text { fird-i/ } \\
\text { fjord/ }\end{array}$ & $\begin{array}{l}\text { fjörður } \\
\text { fjarðar } \\
\text { firði } \\
\text { fjörð }\end{array}$ & $\begin{array}{l}u \text {-Brechung } \\
a \text {-Brechung } \\
\text { urn. Hebung } \\
u \text {-Brechung }\end{array}$ \\
\hline Pl. & $\begin{array}{l}\mathrm{N} \\
\mathrm{G} \\
\mathrm{D} \\
\mathrm{A}\end{array}$ & $\begin{array}{l}*_{f e r p}-\underline{i} u R>f \underline{f i r p}-i u R \\
*_{\text {ferp}}-\bar{o}>-a \\
*_{\text {ferp}}-u m R \\
*_{\text {ferp }}-u n(n)\end{array}$ & $\begin{array}{l}\text { firrd-irl } \\
\text { fjard-al } \\
\text { fjord-um/ } \\
\text { fjord-ul }\end{array}$ & $\begin{array}{l}\text { firðir } \\
\text { fjarða } \\
\text { fjörðum } \\
\text { firði }\end{array}$ & $\begin{array}{l}\text { urn. Hebung } \\
a \text {-Brechung } \\
u \text {-Brechung } \\
u \text {-Brechung }\end{array}$ \\
\hline
\end{tabular}

Die Zahl der isländischen Nominalklassen ist beträchtlich, was zu einem großen Teil auf das Konto dieser (hier nur auszugsweise präsentierten) Kontaktphänomene geht. Zählt man sie extensiv (d.h. ohne Makro- und Mikroklassen zu unterscheiden), so kommt man mit

8 Die späturn. $a$ - und $u$-Brechung besteht in einer Überdiphthongierung mit Akzentumsprung eines urn. kurzen, betonten $e$, das vor $a$ zu ja und vor $u$ zu jo (> nisl. jö) gebrochen wird (genauer: $e>$ *éa > iá > ja) (Iversen 1973: 22). Manche, z.B. Haugen (1982), sehen die $u$-Brechung als eine $a$ Brechung mit $u$-Umlaut. 
Pétursson (1987) auf 60 Klassen.

Fragt man umgekehrt, was auf morphologischem Weg regularisiert wurde, so lassen sich einige wenige Beispiele finden, die gleich hier abgehandelt werden sollen und der Grund dafür sind, dass das Isländische in Abbildung 2 nicht ganz auf den Pol ,Konservierung gerückt wurde, sondern sich auch leicht auf die beiden anderen Achsen ausdehnt: Im Gegensatz zum Deutschen hat sich nie eine Konzentration des $i$-Umlauts auf den Plural ergeben. Schaut man sich z.B. die maskuline $i$-Klasse an, so gilt hier, dass der $i$-Umlaut entweder im gesamten Paradigma generalisiert oder eliminiert wurde: „Der i-Umlaut, der in gewissen Formen [= Gen.(evt. auch Dat.)Sg. + Gen./Dat. Pl. - D.N.] lautgesetzlich fehlte, ist entweder auf alle Formen übertragen oder ganz beseitigt" (Ranke \& Hofmann 1988: 47, ähnlich Nedoma 2006: 48). So erscheint bspw. an. gestr/nisl. gestur 'Gast' komplett umgelautet (im Gegensatz zu nhd. Gast - Gäste), dagegen an. staðr/nisl. staður 'Stelle' komplett unumgelautet (außer dem Dat. Pl. stödum mit $u$-Umlaut). Dabei scheinen (was nicht ausnahmslos gilt) lange Stämme (wie urn. *gast-) eher umgelautet worden zu sein als kurze (wie urn. *stap-) (s. Iversen 1990). ${ }^{9}$ In jedem Fall wurden die Gesamtparadigmen vereinheitlicht (keine grammatische Nutzung des Umlauts) und etwaiger Umlaut lexikalisiert (isl. gestur). ${ }^{10}$ Solche afunktionalen Umlaute bezeichnet Lüssy (1974: 2) als isolierte Umlaute.

Auch bei den Verben wurde der $i$-Umlaut größtenteils dort belassen, wo er eingetreten war, z.B. im Konjunktiv Präteritum der starken Verben (z.B. við fyndum 'wir fänden' zu við fundum 'wir fanden') und dem mancher schwacher Verben sowie in beiden Konjunktiven der zahlreichen schwachen $j$-Verben (zu einem Überblick s. Schnelzer 2008: 26, auch zu neueren Entwicklungen im isländischen Substandard).

Außerdem gibt es nach wie vor eine große Gruppe schwacher sog. Rückumlautverben, z.B. spyrja 'fragen' (vgl. dt. spüren) - spurði - spurt (hier sind es im Gegensatz zu den westgermanischen Sprachen die Kurzstämme, die im Nichtpräsens Nichtumlaut haben). Die beiden Konjunktive enthalten Umlaut. Hier steht also Nichtumlaut im Nichtpräsens Indikativ, ansonsten herrscht überall Umlaut. Als Funktionalisierung ist dies schwerlich zu betrachten.

Wo der $i$-Umlaut tatsächlich eine analogische und kategoriengesteuerte Ausdehnung erfahren hat, ist im Präsens starker Verben mit umlautfähigem Stammvokal: Lautgesetzlich hätte hier - wie bei der deutschen Wechselflexion vom Typ ich fahre, du fährst, sie fährt Umlaut in der 2./3. Ps. Sg. Präs. eintreten müssen. Stattdessen findet er sich heute im gesamten Präs. Sg., also auch in der 1. Ps. Sg., die im Urnordischen auf $-u$ auslautete (und damit $u$ Umlaut bzw. $u$-Brechung hätte auslösen müssen), das im Altnordischen geschwunden ist (s. Tab. 5).

9 Anders und dabei diametral entgegengesetzt zu Dal (1971), Ronneberger-Sibold (1990) und anderen argumentiert dagegen Schulte (1998): Die Kurzstämme (Leichtsilber) seien von der Phonemisierung nicht mehr erfasst worden. Erst die Reduktion des Umlautauslösers („Induktionselement") habe die Phonemisierung der Umlaute bewirkt bzw. initiiert. Nach Langstämmen (Schwersilbern) ist der Auslöser früher geschwunden. Als er dann nach Kurzstämmen schwand, war die Phonemisierungsphase vorüber. Im Altschwedischen findet sich hier allerdings $i$-Umlaut.

${ }^{10}$ Ob hier \pm Umlaut dazu dienen soll, das Gewicht der Stämme anzuzeigen, wie dies Iverson \& Salmons (2009: 99) behaupten, sei dahingestellt bzw. bezweifelt: ,[...] umlaut in Old Norse has become disconnected enough from its phonetic-phonological origins that it takes on the role of reinforcing an extra-morphological determiner of class membership, namely stem weight“. 
Tabelle 5: Die analogische Ausweitung des $i$-Umlauts auf die 1. Ps. Sg. Präsens starker Verben

\begin{tabular}{|c|c|c|c|c|c|}
\hline Num. & Person & $\begin{array}{l}\text { urnordisch } \\
\text { (Endungen) }\end{array}$ & $\begin{array}{l}\text { an. (> neuisl.) } \\
\text { (Stammvokal + Endungen) }\end{array}$ & $\begin{array}{l}\text { nisl. Bsp.: } \\
\text { falla 'fallen' }\end{array}$ & Funktion \\
\hline $\mathrm{Sg}$. & $\begin{array}{l}1 \\
2 \\
1\end{array}$ & $\begin{array}{l}-\mathrm{u} \\
-\mathrm{iR} \\
-\mathrm{iR}\end{array}$ & 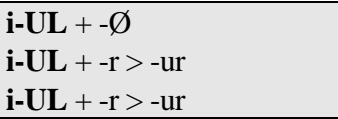 & $\begin{array}{l}\text { fell- } \varnothing \\
\text { fellur } \\
\text { fellur }\end{array}$ & \multirow[t]{2}{*}{$\begin{array}{l}\text { Numerus- } \\
\text { opposition }\end{array}$} \\
\hline $\mathrm{Pl}$. & $\begin{array}{l}1 \\
2 \\
3\end{array}$ & $\begin{array}{l}\text {-ümR } \\
\text {-ið } \\
\text {-an(n) }\end{array}$ & $\begin{array}{l}\mathrm{u} \text {-UL +-um } \\
\text { kein UL + -ið } \\
\text { kein UL + -a }\end{array}$ & $\begin{array}{l}\text { föllum } \\
\text { fallið } \\
\text { falla }\end{array}$ & \\
\hline
\end{tabular}

Schon im Altnordischen ist der analogische Umlaut in der 1. Ps. Sg. vorhanden. Damit konstituiert \pm Umlaut eine Numerusopposition. Ob das sekundäre (junge) $i$ der 2. Ps. Pl. Umlaut bewirken konnte, ist umstritten; zumindest sollte verstärkter gi/ki-Umlaut erwartbar sein, z.B. bei taka: *tekið, der aber nicht vorhanden ist (die Form lautet takið). Im Gegensatz zum Deutschen (vgl. esse - isst) hat die Hebung im Skandinavischen keine Spuren im Verbalbereich hinterlassen. Der $i$-Umlaut wurde nie produktiv - im Gegensatz zum $u$-Umlaut, der noch heute in entlehnten Wörtern als Plural- bzw. (in anderen Flexionsklassen) als Dat. Pl.Marker fungiert: Barðdal et al. (1997: 224) erwähnen z.B. den Schokoladenriegel Mars, isl. mars, der, analog zu dem starken Neutrum land - lönd ('Land - Länder'), den Plural mörs bildet. Dagegen gehört (initialbetontes) banani 'Banane' zur mask. schwachen (im Plural umlautlosen) Klasse, die den Dat. Pl. mit analogischem $u$-Umlaut + Endung -um zu bönunum werden lässt.

Insgesamt ist für das Isländische zu konstatieren, dass der phonologische $i$-Umlaut in erstaunlichem Ausmaß konserviert wurde. Bemerkenswerte Funktionalisierungen sind nicht erkennbar, nur intraparadigmatisch kommt es im Präsens starker Verben mit umlautfähigem Vokal zu einer Numerusopposition, wobei der Umlaut in der unmarkierten Numerusausprägung, dem Singular, sitzt. ${ }^{11}$ Interparadigmatisch breitet sich der Umlaut (entgegen dem Deutschen) nicht aus. Möglicherweise liegt dies an der Vielzahl vokalischer Kontaktphänomene ( $i$ - und $u$-Umlaut, $a$ - und $u$-Brechung, Hebung, Senkung, Labialisierungen) und deren morphologisch ebenso ,ungeordnetem“ Auftreten innerhalb nicht selten eines und desselben Paradigmas, so dass ein mögliches Ziel analogischer Prozesse gar nicht erkennbar war. Nur wenn es ein deutliches Gefälle zwischen möglichst nur zwei Alternanten gibt, ist Analogie erwartbar, d.h. vermutlich greifen morphologische Prozesse eher dann, wenn die Paradigmen nur von einem phonologischen Phänomen durchkreuzt werden (wie beim $i$ Umlaut in den westgermanischen Sprachen; zu dieser Frage s. Abschn. 3). Außerdem besteht oft keine eindeutige Relation des Umlauts zu seinem Basisvokal: Wie in Abbildung 1 gezeigt, können gerundete Umlaute über zwei Wege entstanden sein: Über die Palatalisierung gerundeter Velarvokale oder über die Rundung palataler Vokale. Schließlich kommt gerade für das Isländische hinzu, dass hier - im Vergleich zu allen anderen germanischen Sprachen - der regularisierende Faktor des Sprachkontakts am wenigsten gegeben war.

Bevor wir auf andere Entwicklungen der Umlautprodukte zu sprechen kommen, sei betont, dass alle germanischen Sprachen ein gewisses Maß an Umlautkonservierung aufweisen. Dies

11 Dies widerspricht - übrigens nicht nur hier - dem, was Wurzel (1984) als Domäne und Funktion des Umlauts postuliert. Auch im Neuhochdeutschen befindet sich der Umlaut in unmarkierten Ausprägungen, vgl. die Wechselflexion (s. Abschn. 2.3). 
betrifft die zahlreichen Lexikalisierungen (isolierter Umlaut), d.h. das gesamtparadigmatische Auftreten von Umlaut (vgl. nhd. Sünde, hören, füllen, setzen - nl. zetten, vullen - schwed. sätta, fylla, möta - engl. set, fill, meet). Darin eingeschlossen sind auf Wortbildung beruhende Alternanzen wie z.B. nl./nhd. tam/zahm - temmen/zähmen. Was hier im Mittelpunkt steht, ist der Umgang der Grammatik mit dem Umlaut (funktionaler Umlaut).

\subsection{Eliminierung (Englisch, Niederländisch, Schwedisch)}

Auch für die Eliminierung gilt das eben Gesagte: Alle germanischen Sprachen haben in ihrer Flexion Umlaute getilgt, auch das Deutsche und Luxemburgische. Besonders radikal sind hierbei das Englische und das Niederländische (einschließlich dem Afrikaans) vorgegangen, wobei dies im Englischen durch die allgemein starke Deflexion gefördert wurde. Die letzten Reflexe sind die bekannten irregulären Pluralbildungen vom Typ man - men, goose - geese etc. Weder bei den Verben noch bei den Adjektiven hat sich ein flexivischer Umlautrest erhalten. Das Adjektiv old kompariert heute regulär (old-older-oldest), altes umgelautetes elder kann als weitgehend lexikalisiert betrachtet werden (als Komparativ ist elder/eldest allenfalls noch mit verwandtschaftlichem Bezug verwendbar).

Was das Niederländische betrifft, so gilt nach Goossens (2002b: 221) genau die „Beseitigung des Umlauts in der Verbal- und Nominalflexion“ als einer der konstituierenden Züge bei seiner Herausbildung - daneben übrigens auch die Beseitigung der $e>i$ Hebung in der Verbalflexion: „In de morfologie van de nl. standaardtaal speelt de umlaut zo goed als geen rol. In de flexie is alleen het meervoud steden van stad bekend. Wel zijn er in de woordvorming nog talrijke voorbeelden aan te treffen" (Goossens 2002c: 251). Dabei herrschten bis zu Beginn des Mittelniederländischen (13.-16. Jh.) ähnliche morphologisch distribuierte Umlautalternanzen wie im Deutschen, z.B. Umlaut im Plural von Substantiven (mnl. gast - geste, blat - bleder) und in der Verbflexion (valle - velles/vellet 'falle fällst/fällt'). Noch im Mittelniederländischen werden alle diese Umlaute radikal abgebaut. Die starken Substantive treten langfristig alle in die schwache, traditionell umlautlose Klasse über, d.h. die Plurale geste bzw. bleder wurden zu gasten bzw. bladeren. Auch der erst im Mittelniederländischen aufkommende $s$-Plural war nie mit Umlaut verbunden. Radikale morphologische Klassenzusammenlegungen haben also das Umlautverfahren binnen kurzer Zeit beseitigt. Ähnlich beim Verb: Der Konjunktiv wurde schon früh periphrastisch gebildet, womit der Konjunktivumlaut entfiel, und auch die Wechselflexion hat ein schnelles Ende gefunden. Das (im Neuhochdeutschen noch geltende) Muster mnl. ic valle - du velles, sī/hī vellet - wì vallen, ghì vallet, sī vallen ('fallen') wurde zunächst durch den Ersatz der 2. Ps. Sg. $d u$ durch die 2. Ps. Pl. ghī (heute jij) verändert, denn hierdurch wanderte auch die entsprechende Verbform vallet ins Singularparadigma. Schnell wurde die verbleibende Umlautform sī/hì vellet durch vallet (> valt) ersetzt. In der Adjektivkomparation ist der Umlaut schon früh getilgt worden. Damit zeugt tatsächlich nur noch stad - steden 'Stadt - Städte', trotz schwachen Plurals irregulär umlauthaltig, von diesen einstigen Alternanzen. Eine analogische Ausdehnung des Umlauts hat nie stattgefunden.

Ganz anders verhalten sich diesbezüglich die niederländischen Dialekte, vor allem die östlichen, für die auf die Atlasbände FAND I-III und MAND I und II sowie auf Goossens (2002c) zu verweisen ist. Die folgende Karte zeigt, wie das Umlautaufkommen von Ost nach West abnimmt. 


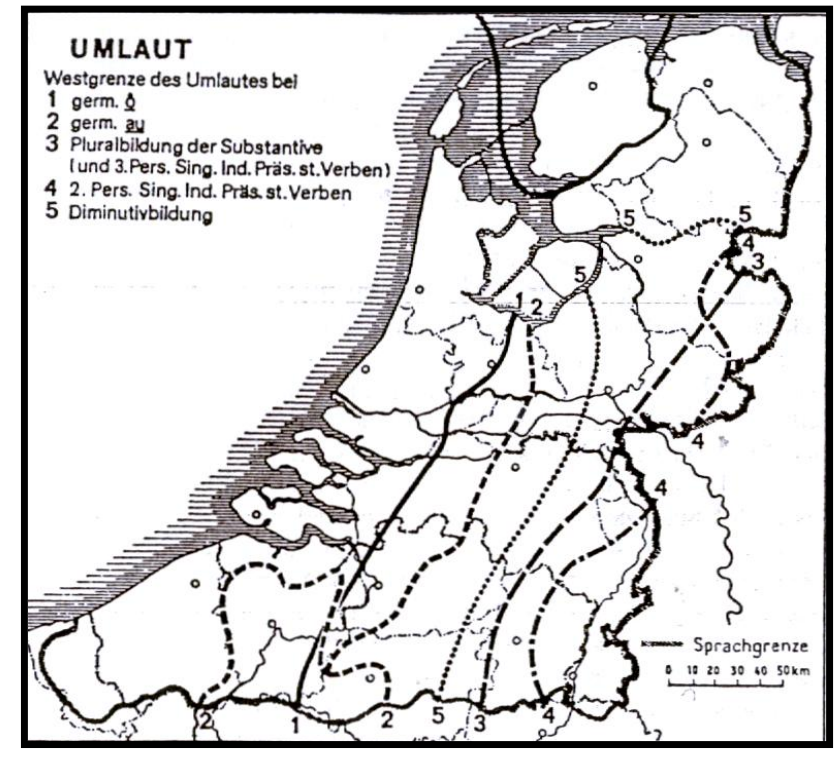

Abbildung 3: Umlautfreundlicher Osten vs. umlautfeindlicher Westen in den niederländischen Dialekten (aus: Goossens 2002a: 207)

Auch das Schwedische (und mit ihm die beiden anderen ostnordischen Sprachen Dänisch und Bokmål) hat den Umlaut schon früh abgebaut, aber nicht so radikal wie das Englische und Niederländische. Die altnordische Numerusopposition im Präsens starker Verben wurde hier komplett ausgeglichen, wobei das Schwedische auch sämtliche Person/ Numerusflexive am Verb eliminiert hat (bspw. lautet das gesamte Präsens von $t a$ 'nehmen' tar, das Präteritum tog). D.h. auch hier ist Deflexion für den Umlautabbau verantwortlich. Dies gilt auch für den Konjunktiv II.

Anders verhält sich dies im Nynorsk, das zusammen mit dem Isländischen und Färöischen zu den westnordischen Sprachen gehört, die den $i$-Umlaut tradiert haben (Haugen 1982: 35): Hier hat sich, genau umgekehrt, die (endungslose) UmlautEinheitsform starker (umlautfähiger) Verben auf das gesamte Präsens ausgebreitet (im Festlandskandinavischen gibt es keine Person/Numerus-Flexion mehr): take 'nehmen' - tek 'Präs.', drage 'ziehen' - dreg, kome 'kommen' - kjem (entrundetes $\emptyset$ ), gjose 'gießen' - gys etc. (s. Beito 1986; zu ihrer Verbreitung s. Barðdal et al. 1997: 321). Hier haben also die einstigen Singularformen gewonnen, indem sie sich ins gesamte Präsensparadigma ausgebreitet haben, allerdings ohne irgendwo mit der Nichtumlautform zu alternieren bzw. zu opponieren. D.h. die Differenz \pm Umlaut wird gar nicht genutzt. Diese Umlautform erhöht allenfalls den Kontrast zum Infinitiv und fügt sich gut in den allgemeinen temporalen Vokalwechsel (Ablaut). Doch wäre (wie in den anderen festlandskandinavischen Sprachen der Fall) auch das unumgelautete Präsens funktionstüchtig, d.h. der Umlaut ist nicht funktionalisiert, er profiliert allenfalls Tempus. 
Dies dürfte auch für ein weiteres (kaum funktionales) Relikt geltend zu machen sein: Im Schwedischen (wieder stellvertretend für die festlandskandinavischen Sprachen) haben sich einige schwache Rückumlautverben erhalten: glädja 'freuen' - gladde 'Prät.', lägga 'legen' - la(de), sätta 'setzen' - satte, sälja 'verkaufen' - sålde, evt. auch säga 'sagen' $s a(d e)$ und göra 'machen' - gjorde. ${ }^{12}$ Hier lässt sich kaum noch ein System erkennen, d.h. der Umlaut (bzw. seine Fortsetzungen) ist als fossiliertes Relikt zu begreifen und profiliert durch idiosynkratische Allomorphie, die fast wie Ablaut wirkt, ebenfalls Tempus. Der Vergleich mit dem Ablaut wird durch die Tatsache gestützt, dass manche dieser Verben auf ihr Dentalsuffix verzichten können (s. die Einklammerungen).

Dagegen hat das Schwedische (mehr oder weniger gilt dies auch für die anderen festlandskandinavischen Sprachen) im Nominalbereich einige Umlaute bewahrt, sowohl in der Adjektivsteigerung (z.B. stor 'groß' - större - störst) als auch in der Substantivpluralisierung (z.B. stad - städer). Dabei sind es ausschließlich hochfrequent vorkommende Einheiten, die diese Relikte bewahren. Eine Endung kommt immer hinzu (von seltenen Ausnahmen wie man - män 'Mann - Männer' abgesehen), d.h. der Umlaut ist nur ein KoPhänomen und damit schwach funktionalisiert. So sind es noch ca. 9 Adjektive und 20-25 Substantive (teilweise mit Pluralschwankungen), die Umlaut konserviert haben und diesen für den Ausdruck markierter Kategorienausprägungen (Plural; Komparativ/Superlativ) einsetzen (Holmes \& Hinchliffe 1994: 18, 108-109; SAG 1999, Bd. II: 72, 198-199). Ob es sich dabei um eine Funktionalisierung handelt, ist fraglich, da der Umlaut nie seine angestammten Klassen verlassen hat. Doch dürfte sein Erhalt durch diese kategorielle Verteilung motiviert bzw. gestärkt worden sein. Dabei alternieren konkret $<\mathrm{a}>$ [a, a:] sowie $<\mathfrak{a}>[0]$ mit $<\ddot{a}>[\varepsilon]$ und $<0>[\mathrm{u},, 0]$ mit $<\ddot{\mathrm{o}}>$ [œ], bei Adjektiven auch [u] mit [y] (zu Beispielen s. Abschn. 1). Klare 1:1-Entsprechungen sind also nicht vorhanden.

Festzuhalten ist, dass der Umlaut im Skandinavischen grammatisch kaum funktionalisiert wurde. Er ist lediglich in manchen Positionen (Plural) verblieben, ohne sich auf weitere Substantive auszudehnen. D.h. sein Erhalt kann teilweise kategoriell motiviert werden, zumal wenn er dort mit Nichtumlautformen alterniert. Ansonsten wurde er, wenn erhalten, lexikalisiert, also auf das gesamte Lexem übertragen (s. Wessén 1992: 20). Auch im Fall der umlautenden starken Präsensformen im Nynorsk ist keine Umlautfunktionalisierung zu erkennen, weil der Umlaut nirgendwo mit Nichtumlaut kontrastiert. Daher kann keine skandinavische Sprache in Abbildung 2 auf der Achse der ,Funktionalisierung' verortet werden. ${ }^{13}$

Generell hat zu den festlandskandinavischen Sprachen hin eine durchgehende Paradigmenhomogenisierung stattgefunden, auch bedingt durch den Wegfall vieler Kasusdistinktionen. Die unter 2.1 für das Isländische nur angedeuteten Verhältnisse (Hebungen, Senkung, Brechungen, verschiedene Umlaute) finden sich hier nicht, die lexikalischen Stämme sind in der Regel uniform (wobei auch Ausgleiche nach der Brechung bzw. den Umlauten hin stattgefunden haben können). Auch der $u$-Umlaut wurde als funktionale

12 Im Isländischen haben sich die Rückumlautverben in großer Zahl erhalten.

13 Mit dieser mangelnden Funktionalisierung des Umlauts dürfte auch seine Verschriftung zu erklären sein: In allen skandinavischen Sprachen wird $/ y(:) /$ als $<y>$ verschriftet, auch wenn sein Basisvokal $/ \mathrm{u}(:) /$ ist (oder war). Basis- und Umlaut-Vokal werden graphematisch also nicht als zusammengehörig markiert, wie dies im Neuhochdeutschen mit $\langle u-\ddot{u}\rangle,<0-\ddot{o}\rangle,\langle a-\ddot{a}\rangle$ und selbst < au - äu> /au - oi/ der Fall ist. 
Einheit stark nivelliert und findet sich nur noch resthaft (vgl. den dän./norw. Plural børn 'Kinder').

Im Vergleich zum Englischen und Niederländischen haben die skandinavischen Sprachen ein stark flektierendes (bzw. agglutinierendes) Nominalsystem nicht nur erhalten, sondern mit der Definitheitsflexion, die keine Auswirkung auf den Umlaut hat, auch ausgebaut. Außerdem hat sich als letzter Kasusrest der Possessivmarker $-s$ erhalten. Auf einen ersten Blick scheint sich ausgeprägte Flexion als solche bereits positiv auf den Erhalt des binnenflexivischen Umlauts auszuwirken. Dabei ist der Umlaut nie alleiniger Träger grammatischer Information, er ist nur ein Ko-Phänomen, indem er immer zu Suffixen hinzutritt. Dies ist anders in Sprachen wie dem Deutschen und Luxemburgischen, wo der Umlaut voll in den Dienst der Grammatik gestellt wird.

\subsection{Funktionalisierung (Deutsch, Luxemburgisch)}

Im Deutschen und Luxemburgischen hat der Pluralumlaut eine ganz andere Qualität erlangt: Hier ist er nicht nur in Verbindung mit den Pluralsuffixen - $e$ und -er in viele bis dato umlautlose Paradigmen eingedrungen - bzw. diese haben das effiziente Umlautverfahren übernommen -, sondern er ist auch zum exklusiven Träger der Pluralinformation avanciert. Damit ist er nicht mehr nur Teil eines diskontinuierlichen Morphems, sondern alleiniger Träger einer grammatischen Information: Der Umlaut ist voll morphologisiert; er ist autonom geworden. Auch bei den Verben und Adjektiven hat er einen hohen Grammatikalisierungsgrad erlangt, wenngleich keine Autonomisierung.

$\mathrm{Zu}$ den Substantiven: Vor allem die Maskulina, aber auch die Neutra nutzen den Umlautplural. Diese Numerusprofilierung ist nach Kazzazi (2004) viel früher als in den Handbüchern üblicherweise angegeben anzusetzen. So muss schon vor althochdeutscher Zeit der Singular der $i$-Klasse in die (umlautlose) $a$-Klasse übergegangen sein - aber eben nur der Singular, um den $i$ - und damit umlauthaltigen Plural umso stärker davon abheben zu lassen. In anderen Klassen (z.B. der iz/az-Klasse) wurden schon im Althochdeutschen die phonologisch entstandenen Umlaute samt stammbildendem Suffix aus den Singularparadigmen (hier: Gen.+Dat. Sg.) analogisch entfernt - Lüssy (1974: 2) nennt dies „funktionellen Rückumlaut“ -, noch bevor der Umlaut im Plural phonemisiert oder morphologisiert war: Auch als Allophon, in Verbindung mit einem $i$-haltigen Suffix, hat der Umlaut offensichtlich seine Wirkung entfaltet. ${ }^{14}$ Nur so ist erklärbar, dass Wurzel- und $u$-Stämme wie ahd. fuoz 'Fuß' schon früh in die $i$-Klasse übergetreten sind (später folgen auch viele $a$-Stämme). Nach Ronneberger-Sibold (1990: 198) war der Umlaut also schon in dieser Phase ,an der morphologischen Funktion der Pluralanzeige beteiligt““.

14 Konkret: Der frühahd. Singular lamb, lembires, lembire, lamb wurde noch im Altoberdeutschen homogenisiert zu lamb, lambes, lambe, lamb. - Selbst in Paradigmen (wie den schwachen Maskulina), die keinen $i$-haltigen Pluralmarker hatten, wurden die Umlautformen schon früh aus dem Singular beseitigt (funktionell rückumgelautet), vgl. frühes Althochdeutsch Nom.-Akk. Sg. namo, nemin, nemin, namun > ahd. namo, namin, namin, namun (s. auch Robinson 1980). 
Damit sind ahd. die kurzsilbigen wie die langsilbigen ehemaligen $i$-Stämme im Singular analogisch den $a$-Stämmen gleich geworden. Im Plural dagegen sind bei den $i$-Stämmen die lautgesetzlichen, umgelauteten Formen erhalten geblieben, so dass hier nun ein Muster 'unumgelauteter Singular : umgelauteter Plural' gast : gesti existiert. Dieses Muster hat sich jedoch nicht zufällig ergeben, sondern es ist offenbar über einen längeren Zeitraum hinweg regelrecht darauf hingearbeitet worden, den Umlaut dort zu eliminieren, wo er dieser angestrebten Verteilung im Weg ist. Und nur das Althochdeutsche geht diesen Weg in solcher Konsequenz. (Kazzazi 2004: 152).

Das heißt, schon im Voralthochdeutschen konnte Umlaut zwar nur lautgesetzlich entstehen, er konnte aber schon morphologisch beseitigt werden (Kazzazi 2004: 153). Und er konnte durch die (morphologische) Übernahme $i$-haltiger Pluralendungen (wie z.B. bei den Wurzelnomina geschehen) aus anderen Paradigmen (phonologisch) erzeugt werden. Während auch das Altenglische die Umlautbeseitigung im Singular praktiziert, divergiert es stark vom Althochdeutschen, indem es die $i$-Stämme auch im Plural nach den zahlreicheren (umlautlosen) $a$-Stämmen ausgleicht. Hier trennen sich also - in den Worten von Kazzazi (2004) - die Wege.

Die neutrale iz/az-Klasse, die im frühen Althochdeutschen aus gerade einmal neun Mitgliedern bestand und Lebewesen rund um die menschliche Siedlung enthielt, hat noch im Althochdeutschen Zuwächse erfahren. Alt scheint nach Braune \& Reiffenstein (2004: 188) ahd. kalb, lamb, huon, hrind, farh 'Ferkel', ei, luog 'Tierlager', hrīs 'Reis, Spross' und blat 'Blatt' zu sein. Hinzu kommen noch im Althochdeutschen feld (zunächst noch mit dem gehobenen Plural fildir), har 'Haar', hol 'Höhle', loub 'Laub', krūt 'Kraut', brët, rad, grab, holz, loh, hūs, wëlf 'Junges', lid 'Glied' etc., wobei es hier noch die umlautauslösende irEndung ist, die übertragen wird. (ebd.). Nach mittelhochdeutscher Zeit weitet sich das mittlerweile zu ,Umlaut $+-e r^{6}$ mutierte Verfahren analogisch weiter aus, auch auf Maskulina (vgl. Mann - Männer). Heute enthält diese Klasse ca. 100 hochfrequente Neutra und Maskulina. Keine andere germanische Sprache außer dem sprachhistorisch eng verwandten Luxemburgischen hat diese Klasse so stark ausgebaut (in den anderen Sprachen ist sie i.d.R. gar nicht mehr vorhanden). Grund dafür ist das saliente Pluralverfahren. ${ }^{15}$

Kazzazi (2004) macht für diesen deutschen Sonderweg eine besonders lange Phase allophonischer Alternanz verantwortlich, die einen „breiteren Spielraum für Analogie lässt““ (158), etwas salopp formuliert: Es konnte mehr ausprobiert werden. Bekanntlich findet die Nebensilbenabschwächung erst auf dem Weg vom Alt- zum Mittelhochdeutschen statt, also etwa im 10. Jh., wobei hier eine Nord-Süd-Staffelung besteht, d.h. im Alemannischen erfolgt die Schwächung deutlich später. Im Vergleich zu allen anderen Sprachen währt diese Phase hier am längsten.

15 Von dieser frühen Umlautfunktionalisierung weichen die starken Feminina ab, die im Gen./Dat. Sg. bis ins Mittelhochdeutsche, teilweise sogar ins Frühneuhochdeutsche hinein den Umlaut bewahren (den sie außerdem im Plural enthalten). Erst spät wird der Singular vom Umlaut bereinigt (Paul 2007: 195-198). Steffens (2010) weist für das Mainzer Frühneuhochdeutsche nach, dass der Umlaut im Dat. Sg. deutlich vor dem Gen. Sg. beseitigt wird. Warum die starken Feminina diesen Sonderweg gehen, könnte an der (bis dato noch zu wenig untersuchten) Genusprofilierung liegen, die u.a. über verstärkte Divergenzen im Flexionsklassenverhalten realisiert wird. 
Die mittelhochdeutsche Nebensilbenabschwächung sorgte für eine weitere Stärkung des Umlauts, nicht weil sie seine längst erfolgte Phonemisierung offenlegte, sondern die Weiche für den reinen Pluralumlaut ohne Endung stellte. Damit wurde die Funktionalität des Umlauts massiv erhöht, er wurde als grammatischer Pluralmarker autonom. Grundlage hierfür sind zweisilbige Maskulina der $i$-Klasse, da ein Schwa in der 3. Silbe aus prosodischen Gründen besonders schwundanfällig war: ahd. apful - apfuli 'Apfel - Äpfel' $>$ apful - epfili > mhd. apfel - epfele > apfel - epfel (e-Apokope) > nhd. Apfel- Äpfel. Der Umlaut war dabei längst zu einem Index für das Plural-ə geworden, da er es ja anfänglich zwingend voraussetzte (während umgekehrt nicht jedes Plural-ə einen Umlaut voraussetzte, s. mhd. tage < ahd. taga). Dies führte letztlich zu einer leichteren Verzichtbarkeit des Schwa. In der Folge haben zahlreiche zweisilbige Maskulina aus anderen Klassen diesen morphologischen $i$-Umlaut analogisch übernommen, z.B. Schnäbel, Väter, Brüder, Gärten, Öfen, Bögen, Schäden etc., ja sogar zwei Feminina: Mütter und Töchter. Noch heute schwanken einige Maskulina im Plural: Wagen/Wägen, Hammer/Hämmer, Ranzen/Ränzen, Haufen/Häufen. Bezeichnenderweise sind die Umlautplurale eher süddeutsch.

Auch die Einsilber mit Umlaut $+-e$ im Plural haben stark zugenommen und Mitglieder selbst aus der schwachen Klasse gewonnen (z.B. die hanen > die Hähne). Umlautende Plurale sind also im Deutschen omnipräsent und stärken seinen introflektierenden Charakter. Hier kommt es jedoch - im Gegensatz zum Luxemburgischen und zum Alemannischen - nicht zu umlautenden Einsilbern: Im deutschen Standard gilt als Outputbeschränkung für Plurale Zweisilbigkeit (Trochäen).

Das Luxemburgische schießt gleich in mehrfacher Hinsicht weit über das Deutsche hinaus (s. hierzu Nübling 2005, 2006): Erstens hat es den Pluralumlaut noch stärker ausgebaut. Weitaus mehr Substantive (meist Maskulina) lauten um, darunter auch schwache: lux. Aarm - Äerm 'Arm - Arme', Rass - Rëss 'Riss - Risse', Stach - Stéch 'Stich Stiche', Mount - Méint 'Monat - Monate', Rouer - Réier (f.) 'Röhre - Röhren', Numm Nimm 'Name - Namen'. Zweitens - dies zeigen diese Beispiele - lauten auch zahlreiche Einsilber um, d.h. die für das Deutsche bestehenden Outputbeschränkungen gelten hier nicht. Drittens ist, wie oben bereits erwähnt, das einstige System der 1:1-Entsprechung zwischen Umlaut- und Basisvokal in beide Richtungen durchbrochen. Der Vergleich des Singular-Plural-Vokalismus im Deutschen und Luxemburgischen in Abbildung 4 macht dies schlagartig deutlich. Während im Deutschen nicht nur transparente 1:1-Beziehungen und damit vorhersagbare Relationen bestehen, sondern auch noch der Singularvokal velar und der Pluralvokal palatal ist, hat das Luxemburgische beides durchbrochen: Quantitativ wie qualitativ tun sich hier viele Asymmetrien auf, die Abbildung 4 illustriert. Die dicken Linien bezeichnen dabei sehr häufige Alternanzen, die dünnen seltenere, die jedoch alle den Terminus Umlaut synchron nicht mehr rechtfertigen: Durch die nicht mehr gegebene Vorhersagbarkeit des Pluralvokals aus dem Singularvokal und durch die partielle Aufhebung der [ \pm palatal]-Opposition ${ }^{16}$ ist der Umlaut demotiviert, weitgehend arbitrarisiert und damit dem Ablautverfahren synchron nahegekommen.

\footnotetext{
${ }^{16}$ Im Singular befindet sich auch [e:], das im Plural mit [ع] alterniert.
} 

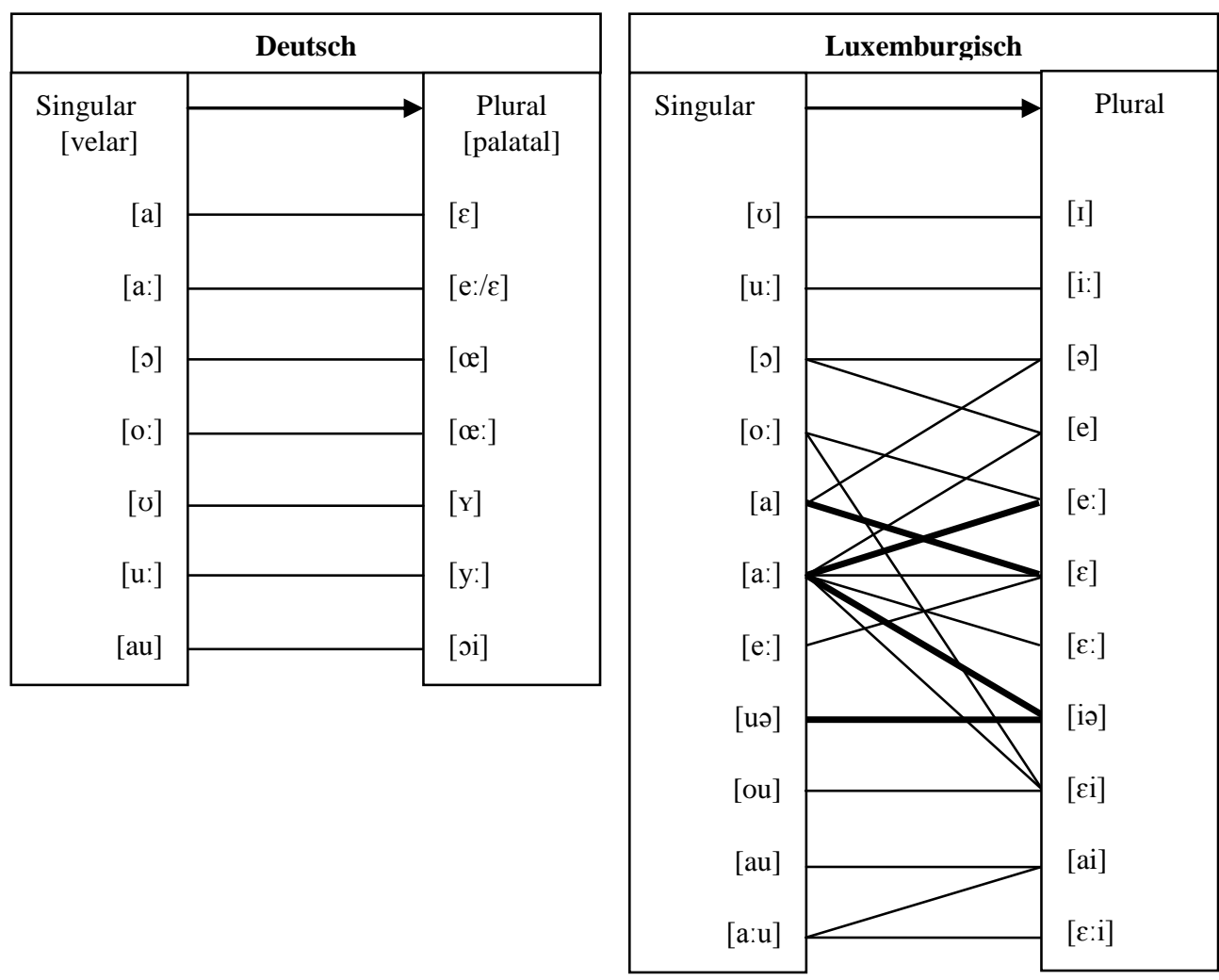

Abbildung 4: Erhalt vs. Beseitigung der 1:1-Relation zwischen Basisvokal und Umlaut im Deutschen vs. Luxemburgischen

Außerdem - viertens - sind viele Substantive erst spät zu diesem Alternanzverfahren gekommen, da sie historisch nie einen Velar besessen haben, z.B. Rass < Riss 'Riss' (mit dem Pl. Rëss). Erst nach der $i>a$-Senkung konnte dieses Verfahren greifen. ${ }^{17}$ Auch werden noch heute auffällig viele Lehnwörter modifikatorisch pluralisiert, vgl. Mount - Méint 'Monat(e)', Club - Clib 'Club(s)', und bei dem initialbetonten Gallizismus Tirang - Tiräng 'Schublade(n)' spielt sich die Alternanz im unbetonten Vokal ab. Mit der verstärkten Integration der Numerusinformation in die lexikalische Wurzel (alleiniger Pluralmarker, viel Allomorphie, hohe Typenfrequenz) korrelieren übrigens auch konsonantische Modifikationen, für die auf Nübling $(2005,2006)$ verwiesen sei. Tatsache ist, dass das Luxemburgische damit das Grammatikalisierungsmaximum innerhalb der germanischen Sprachen erreicht hat.

Auch in der deutschen Adjektivkomparation versieht der Umlaut morphologische Funktionen und hat sich, nach der Nebensilbenabschwächung, aus seinen historischen Bedingungen gelöst, indem er aus bestimmten Adjektiven getilgt und in andere über-

17 Vergleichbares beschreibt Götz (1996) für das Bairische. 
nommen wurde. Dabei spielte die Tokenfrequenz des Adjektivs eine entscheidende Rolle, d.h. hier wird das kürzere, wenngleich schwierigere Verfahren präferiert (zu Näherem s. Augst 1971, Nübling et al. ${ }^{2} 2008:$ 216-219). Dies gilt weniger für das Luxemburgische, da es den Komparativ analytisch mit méi + Adj. bildet.

Bei den starken Verben findet sich im Deutschen bis heute Umlaut in der sog. Wechselflexion, wobei hier die $a>e$-Umlaute dominieren: fahre - fährst/fährt; daneben: stoße - stößt, saufe - säufst/säuft ( $u>\ddot{u}$-Umlaut ist inexistent). Insgesamt gibt es noch 19 jeweils hochfrequente Umlaut-Verben, die dieses funktional schwer zu rechtfertigende Relikt bewahrt haben (hinzu kommen 36 Verben mit $e>i$-Hebung). ${ }^{18}$ Im Gegensatz zur Pluralbildung tritt der Umlaut nur zur Endung hinzu. ${ }^{19}$ Ganz anders im Luxemburgischen, wo es zwar auch nur ca. 55-60 Wechselflexionsverben gibt, doch wo es zu mehr als 20 verschiedenen Wechselflexionsmustern kommt (inklusive einstiger Hebung) und wo auch schwache und frühere Wurzelverben (wie 'machen', 'haben', 'sagen', 'gehen', 'tun') die 2. und 3. Sg. umlauten bzw. alternieren lassen, denn ähnlich wie bei der Pluralbildung tut sich hier wieder ein ziemliches ,Wirrwarr' bei den Zuordnungen von Basis- und Wechselflexionsvokalen auf (zu Details s. Nübling 2001). Während das Deutsche hier phonologischen Umlaut konserviert hat, hat das Luxemburgische von analogischen Verfahren reichen Gebrauch gemacht, selbst bei den schwachen und den Wurzelverben, die historisch keine Wechselflexion aufbauen konnten.

Im Deutschen hat sich beim Konjunktiv II eine echte Umlautmorphologisierung eingestellt. Die Ableitungsbasis der Konjunktivform war ursprünglich die 3. Ablautstufe. Als nach dem präteritalen Numerusausgleich im Frühneuhochdeutschen in manchen Ablautreihen die 3. Stufe zugunsten der 2. Stufe aufgegeben wurde, ist der damals phonologisch schon lange nicht mehr produktive Umlaut morphologisch auf den neuen Präteritalvokal projiziert worden: ich trank/wir trunken $\rightarrow$ ich trünke/wir trünken $>i$ ich/wir trank(en) $\rightarrow$ ich tränke/wir tränken. Auch hier ist es so, dass der Umlaut zwar morphologisiert, aber in manchen Positionen nur Teil des diskontinuierlichen Konjunktivausdrucks ist (sieler tränk $\underline{e}$ ), in anderen dagegen allein den Konjunktiv markiert (wir/sie tränken, ich tränke). Allerdings hat der Konjunktivumlaut nie seine angestammte Domäne der starken Verben verlassen, sieht man von den beiden schwachen Verben haben (hätte) und brauchen (bräuchte) ab. Damit wird deutlich, dass die Umlaut-Funktionalisierung nicht mit interparadigmatischer analogischer Ausdehnung einhergehen muss (Konjunktiv), es aber kann (Plural). Im Luxemburgischen gibt es dagegen kaum noch synthetische Konjunktive.

Nach den beiden beschriebenen, über die bloße Funktionalisierung hinausgehenden Umlautentwicklungen der Autonomisierung und Arbitrarisierung lässt sich als weiterer (und letzter) Durchbruch eine Transkategorialisierung des Umlauts feststellen: Bis heute gilt der Umlaut im Präsens Plural (und, davon abgeleitet, im Infinitiv) der vier nhd. Präteritopräsentien können, mögen, dürfen und müssen als ungeklärt. Eine lautgesetzliche Basis kommt ihm nicht $\mathrm{zu}$, und auch alle in der traditionellen Literatur bisher vorgebrachten Erklärungen, die in Nübling (2008) resümiert werden, vermögen nicht die Frage zu beantworten, weshalb er ausgerechnet im Plural und nicht auch im Singular

18 Vgl. hierzu auch Wurzel (1984).

19 Nach Dal (1971) hat sich der Umlaut nur deshalb beim Verb erhalten, weil dieses ohnehin ein alternierendes Verfahren, den Ablaut, kultiviert. 
vorkommt. In Anbetracht dessen, dass es - genau wie im Nominalbereich - die Pluralkategorie ist, die hier umlautet, aber auch der Tatsache, dass transkategoriale Marker auch in anderen Sprachen (wie dem Arabischen und Türkischen) vorkommen und dort wiederum zuvörderst die Numeruskategorie symbolisieren, habe ich in Nübling (2008) dafür argumentiert, auch für das Deutsche Transkategorialität anzunehmen: Die Numerusinformation 'Plural' macht im Nominal- und Verbalbereich vom gleichen Verfahren, dem Umlaut, Gebrauch und realisiert damit eine Art transkategoriale Uniformität. Für diese Erklärung sprechen auch chronologische Fakten (die Entstehung beider morphologischen Umlaute im Mittelhochdeutschen) sowie dialektale: Die Hochburg morphologisierter Umlaute, nämlich die Deutschschweiz, kultiviert nicht nur bei einigen Präteritopräsentia, sondern auch in der Sonderkleinklasse der sog. Kurzverben Umlaut im Plural. ${ }^{20}$ Dies zeigt Tabelle 6. Zu Details und der genauen geographischen Verbreitung der Verbalpluralumlaute s. Fischer (1989), Weber (1987) und den SDS III.

Tabelle 6: Verbale Umlautplurale bei Kurz- und Modalverben im Luzerndeutschen nach Fischer (1989: $\S 197-199)^{21}$

\begin{tabular}{|c|c|c|}
\hline $\begin{array}{l}\text { Kurzverben } \\
\text { (Infinitiv) }\end{array}$ & 1.-3. Singular Präsens & $\begin{array}{l}\text { 1.-3. Plural Präs. (Osten) } \\
\text { (Einheitsplural auf }-n d)\end{array}$ \\
\hline 1) gòò 'gehen' & gòò(ne), gòòsch, gòòt & gönd \\
\hline 2) stòò 'stehen' & stòò(ne), stòòsch, stòòt & stönd \\
\hline 3) lòò 'lassen' & lòò(ne), lòòsch, lò̀t & lönd \\
\hline 4) schlòò 'schlagen' & schlòò(ne), schlò̀sch, schlòòt & schlönd \\
\hline 5) aafòò 'anfangen' & fò̀̀(ne), fò̀ssch, fò̀t aa & fönd $a a$ \\
\hline 6) chò̀ 'kommen' & chome, chouscht, chont & chömid \\
\hline 7) haa 'haben' & ha(a), hëscht, hët & hënd \\
\hline 8) nää 'nehmen' & neme, nemscht, nemt & nänd \\
\hline 9) tue 'tun' & tue(ne), tuescht, tuet & tüend \\
\hline Modalverben & 1.-3. Singular Präsens & 1.-3. Plural Präsens \\
\hline 1) chönne 'können' & cha, chauscht, cha & chönid \\
\hline 2) möge 'mögen' & mag, magscht, mag & mögid \\
\hline 3) müesse 'müssen' & mиеs, mиеscht, тиеs & müend \\
\hline 4) wëlle 'wollen' & $\begin{array}{l}\text { well, wellscht, well } \\
\text { wòtt, wòttscht, wòtt }\end{array}$ & wënd \\
\hline
\end{tabular}

Weitere Argumente pro Transkategorialität sind in Nübling (2008) aufgelistet. Worin die verbalen Pluralumlaute jedoch die nominalen Umlaute übertreffen, ist - so zumindest bei den nhd. Präteritopräsentia, aber auch bei manchen der schweizerdt. Verben - die Loslösung von ihrem Basisvokal, d.h. die Reduktion auf das Merkmal der bloßen Palatalität ohne Bezug zum Basisvokal: In kann - können, mag - mögen sowie in darf - dürfen alterniert (im Gegensatz zu muss - müssen) der Umlautvokal nicht mit seinem üblichen

20 Auch im Niederdeutschen kommt es im Präteritum Plural starker Verben zu (bis dato nicht geklärten) Umlautungen, s. Schirmunski (1962: 526-528).

${ }^{21}$ Teilweise konnten hier nicht alle Diakritika, insbesondere über Umlautgraphemen, übernommen werden (s. Fischer 1989: §199). 
Korrelat. Hier liegt - in gewisser Weise vergleichbar mit dem Luxemburgischen - eine Arbitrarisierung vor, wobei der Pluralvokal sich in allen Fällen das Merkmal der Palatalität teilt (was im Luxemburgischen mit dieser Ausnahmslosigkeit nicht mehr gilt). D.h. hier hat sich die Information 'Plural' auf ein phonologisches Merkmal, [+palatal], zurückgezogen. Die Segmentalität reduziert sich dadurch noch stärker, außerdem ist das Alternanzprinzip mit dem Basisvokal durchbrochen. Die Reduktion morphologischer Informationen auf das Merkmal [+palatal] stellt auch Moulton (1971) für die Schweiz fest, wobei das Alternanzprinzip mit dem Basisvokal intakt bleibt (s. hierzu den folgenden Abschn. 3; s. auch Chapman 1994).

Schematisch lassen sich die drei Weiterentwicklungen des funktionalen Umlauts: Autonomisierung, Arbitrarisierung und Transkategorialisierung, mit Abbildung 5 darstellen: Während die morphologische Autonomisierung auf der gleichen Ebene wie die Funktionalisierung liegt und von dieser eine Radikalisierung bildet (der Umlaut allein besitzt morphologischen Status und kooperiert nicht mit einem Affix), setzen Arbitrarisierung und Transkategorialisierung nicht zwingend die Autonomisierung voraus (vgl. lux. Wuert - Wierder 'Wort - Wörter'). Im Fall von lux. Numm - Nimm 'Name Namen' liegt jedoch beides vor: Autonomisierung und Arbitrarisierung. Die Transkategorialisierung setzt die Funktionalisierung voraus (vgl. nhd. muss - müssen), arbeitet aber typischerweise mit arbitrarisierten Umlauten (darf-dürfen, kann - können).

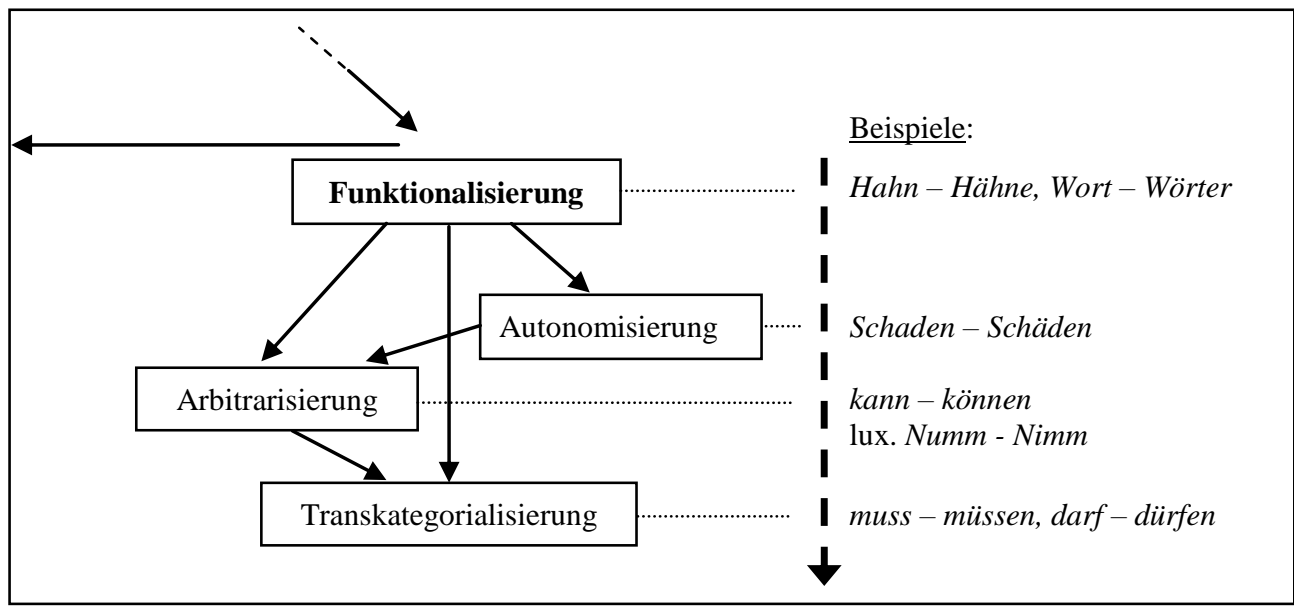

Abbildung 5: Autonomisierung, Arbitrarisierung und Transkategorialisierung des Umlauts 
3. Was bedingt die Funktionalisierung?

Der Fall Schweizerdeutsch: Phonologische Umlautfeindlichkeit und morphologische Umlautfreundlichkeit

Abschließend soll die Frage beantwortet werden, wie die optimalen Bedingungen für eine grammatische Nutzung des Umlauts aussehen. Man sollte vermuten, dass da, wo eine Fülle an phonologischen Alternanzen vorhanden ist, auch die meisten Funktionalisierungen zu erwarten sind, da hier das ,phonologische Angebot' und damit die Auswahl am größten ist. Diese Hypothese wurde bereits anhand des Isländischen verworfen: Hier haben neben verschiedenen Umlauten viele weitere vokalische Kontaktphänomene gewirkt - eine Funktionalisierung hat dagegen kaum stattgefunden. Bewegen wir uns vom Nordrand der Germania an ihren Südrand, erlangt man Evidenz für die umgekehrte Annahme, dass es eher ein möglichst geringes phonologisches Angebot ist, das zu Morphologisierungen führt: Das Schweizerdeutsche weist eine auffällig starke Umlautmorphologisierung auf, Moulton (1971: 16) spricht sogar „Umlautwucherung“. ${ }^{22}$

Abbildung 6 zeigt zunächst anhand einer Verbreitungskarte von Familiennamen mit $<0>$ vs. <ö>, dass der Süden hier keinen Umlaut kennt: Die dunkel eingefärbten Gebiete bzw. Kreisdiagrammanteile bezeichnen die umlautlosen Namen, die sich nach Süden in die Schweiz fortsetzen und verdichten.
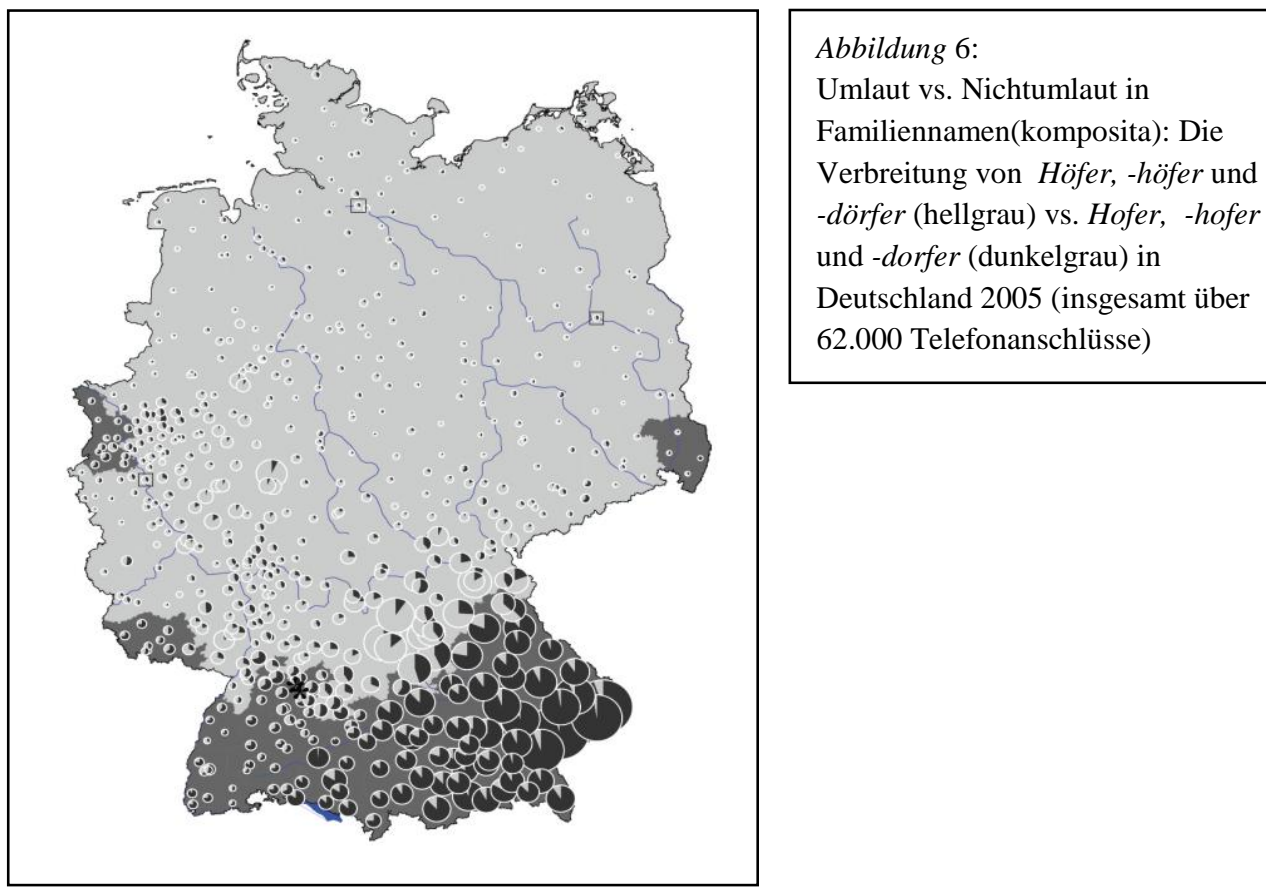

Umlaut vs. Nichtumlaut in

Familiennamen(komposita): Die

Verbreitung von Höfer, -höfer und

-dörfer (hellgrau) vs. Hofer, -hofer und -dorfer (dunkelgrau) in

Deutschland 2005 (insgesamt über 62.000 Telefonanschlüsse)

22 Dabei erweist sich nach Lüssy (1974) die Nordostschweiz als das Kerngebiet des funktionalen Umlauts, weniger der südliche Teil der Deutschschweiz. 
Diese Karte (in Abb. 6) weist die phonologische Grundlage der Umlautung vor dem Derivationssuffix -er < lat. -ārius aus, das üblicherweise Umlaut bewirkt hat (Antonsen 1969: 205, Lüssy 1983: 1086). Dabei gilt auch für die anderen Umlaute ein klarer Nord/Süd-Gegensatz, auch wenn sich im Detail andere Linienverläufe zeigen (s. hierzu Kunze \& Nübling 2009: 2-27, 174-204, 290-325). Die oberdeutsche Umlautfeindlichkeit ist in der Dialektologie ein bekanntes Faktum ${ }^{23}$ und begründet sich u.a. mit der stärkeren Wirkung umlauthindernder Konsonanten (meist Velare, s. hierzu Iverson \& Salmons 2000). Nach Sonderegger (1959: 6) nimmt diese von Norden nach Süden hin zu bzw. die Fähigkeit, Konsonantenwiderstände zu überwinden, ab: Die Umlautwirkung ist im Norden der Germania am stärksten ausgeprägt (s. Abb. 7). Allerdings ist in der Literatur umstritten, ob die oberdeutsche Umlautfeindlichkeit nur auf umlauthemmender Konsonanz (oder frühem Ausfall der Umlautauslöser) beruht oder bzw. und ob hier auch morphologische Faktoren gewirkt haben (Ausgleich einstiger umlautender Paradigmen; zu dieser Diskussion s. Antonsen 1969).

\begin{tabular}{|ll|}
\hline Sprache & Umlauthinderung \\
Altnordisch & keine \\
Altenglisch & keine \\
Altfriesisch & gering (vor $r+$ Kons.) \\
Altsächsisch & gering (vor $h l, h n, h s, h t$, z.T. $r+$ Kons.) \\
Mittelniederdt. & gering \\
Mittelniederländ. & nicht häufig (z.T. $h t$, z.T. $r)$ \\
Niederländisch & vorhanden $(h h,(c) h t)$ \\
Fränkisch & vorhanden $(h t, h s$, Kons. $+w)$ \\
Oberdeutsch & stark ausgeprägt $(h t, h s, h h, c h, c k$, Kons. $+w$, \\
& $\quad l$ - und $r$-Verbindungen) \\
\hline
\end{tabular}

Abbildung 7: Die Zunahme von Umlauthinderungen von Norden nach Süden (nach Sonderegger 1959: 6)

Paradoxerweise ist jedoch die morphologische Nutzung des Umlauts im Süden der Germania am höchsten (wozu man auch das Luxemburgische zählen kann): Viel mehr als in der deutschen Standardsprache werden im Alemannischen Substantive, insbesondere Maskulina der traditionell umlautlosen schwachen Klasse und der starken $a$-Klasse, im Plural umgelautet (Name - Näme, Brunne - Brünne, Aarm - Ä̈rm, Hund - Hünd), auch gegenwärtig (Apparaat - Apparäät, Final - Finäl), d.h. dieses Verfahren ist produktiv (Lüssy 1974: 62ff.). Oft wird als Katalysator die stärker wirkende $e$-(und $n$ )-Apokope gesehen, die einen rein modifikatorischen Plural erzwungen habe (z.B. Lüssy 1983: 1086). Bei den Adjektiven erreicht der Umlaut in Komparativ und Superlativ den Rekord, zumal hier die für das Standarddeutsche geltende Inputbeschränkung der Einsilbigkeit durchbrochen ist, vgl. z.B. luschtig - lüschtiger, mager - mägerer, trurig - trüriger, langsam lengsemmer. Bei den Verben hat sich die umlautende Wechselflexion nicht durchgesetzt

${ }^{23}$ Hierzu s. Sonderegger (1959), Schirmunski (1962), Antonsen (1969), Lüssy (1974, 1983). 
(von kleinen Gebieten, z.B. Simmental, abgesehen): alem. ich fahr, du fahrsch, er/sie fahrt. Im Konjunktiv II gelten komplizierte, aber tendenziell (zumindest in bestimmten Ablautreihen) umlautlose Verhältnisse, wobei der Umlaut hier abgebaut wurde (sog. „Rückumlaut" nach Lüssy 1974). ${ }^{24}$,Dafür" hat sich bei den in Tabelle 6 aufgeführten Kurzund Modalverben ein lautgesetzlich nicht motivierbarer Umlautplural etabliert, den wir als transkategorialen Marker interpretiert haben: Ein salientes nominales Pluralbildungsverfahren wurde auf eine Kleingruppe besonderer und vor allem hochfrequenter Verben übertragen.

Moulton (1971) beschreibt mehrfach, wie äußerst schwach besetzte Alternanzen anscheinend zur Vorlage für analogische Übertragungen und Morphologisierungen avancierten. So konnte sich z.B. zu germ. /o/, das nur auf gesenktes /u/ rückführbar ist, per definitionem kein /ö/ entwickeln, weil ein nachfolgender Palatal die Senkung von /u/ > /o/verhindert hätte, vgl. noch frühahd. loh - luhhir > ahd. loh - lohhir 'Loch Löcher'. Noch im Althochdeutschen hat der Plural einen Stammvokalausgleich zum Singular vorgenommen. Dabei gab es nicht viele Singulare mit kurzem /o/. Dennoch hat sich hier eine Morphologisierung ergeben, die gerade in der Schweiz am ausgiebigsten genutzt wird. Auch andere Oppositionen (im Alemannischen gibt es weitaus umfangreichere Vokalsysteme als im Standard) sind lautgesetzlich nur selten, manchmal sogar überhaupt nicht entstanden, finden sich aber in morphologisierten Alternanzen. Moulton (1971) schließt daraus, dass es nur das Merkmal [+palatal] sein kann, das eine Morphologisierung erfahren hat und damit analogisch übertragbar wurde, und nicht etwa konkrete \pm Umlaut-Alternanzen (s. auch Chapman 1994).

Die morphologisierte und heute noch produktive Merkmalopposition [-palatal] $\leftrightarrow$ [+palatal] wurde auch auf Vokale projiziert, die im Mittelhochdeutschen noch nicht [-palatal] waren, z.B. mhd. [ei], das in manchen Schweizer Dialekten zu [a:] monophthongiert wurde (in anderen zu [o:] oder [o:ə]), z.B. mhd. breit > alem. brāt. Zu diesen neuen Velarvokalen haben sich (ähnlich wie im Luxemburgischen) sekundär morphologische Umlaute gebildet, z.B. zu brāt br[æ:]ter 'breiter' (bzw. br[œ:]ter und $b r[œ: 2] t e r)$. Doch wurden mögliche Arbitrarisierungen (in der Regel) im Keim erstickt: So wurde mhd. langes /a:/ in weiten Teilen der Schweiz lautgesetzlich zu /o:/ verdumpft, nicht aber das Umlautprodukt /æ:/. Dieses hat, um die asymmetrische Alternanz */o:/ $\leftrightarrow$ /æ:/ zu vermeiden, daraufhin eine analogische Verdumpfung zu /œ:/ vollzogen, vgl. alem. Pfohl Pföhl 'Pfahl - Pfähle' (zu Details s. Moulton 1971, zu ähnlichen Phänomenen im Bairischen Götz 1996). Allerdings übersieht die Forschung, dass bei den verbalen Pluralumlauten (s. Tab. 6) diese Asymmetrie geduldet wird (mag - mönd 'mag - mögen', chunt - chönd 'kommt - kommen'), sogar die Durchbrechung der Opposition [-palatal] [+palatal]: nint - nänd 'nimmt - nehmen', will - wänd 'will - wollen'.

24 Man kann pauschal sagen, dass $\ddot{u}$ zu $u$ rückumgelautet wurde (würd(e) > wurd, stürb(e) > sturb), während sich $\ddot{a}$ als Konjunktivmarker erhalten hat (gäb, näm, chäm, tät). Dieses $\ddot{a}$ hat sich sogar analogisch ausgebreitet: mäch 'würde machen', zäch 'zöge', bläb ‘bliebe' etc. (s. Lüssy 1974).

25 Besonders im Zürichdeutschen kommt es auch im Nominalbereich zu asymmetrischen Umlautverhältnissen, vgl. etwa Taarm - Tëërm [ع:] 'Darm - Därme', Chraage - Chrääge [æ:] 'Kragen Krägen', Naat - Nööt [œ:] 'Naht - Nähte' (s. Weber 1987: 62-65). Dies stützt entsprechende Verhältnisse im Verbalbereich. 
Soweit die Verhältnisse am Südrand der Germania. Dieses instruktive Beispiel für phonologische Umlautfeindlichkeit bei gleichzeitiger morphologischer Umlautfreundlichkeit deutet darauf hin, dass es eher ein geringes phonologisches Angebot ist, das den Nährboden für Funktionalisierungen bildet. Das dies bestätigende Komplement bildet das Nordgermanische (v.a. Isländisch), das trotz phonologischer Umlautfreundlichkeit morphologische Umlautfeindlichkeit an den Tag legt.

\section{Fazit}

Nur konsequent betriebener Sprachwandelvergleich gerade dicht verwandter Sprachen zeigt und erklärt, wie und weshalb ähnliche Voraussetzungen zu grundlegend unterschiedlichen Resultaten führen können. Mit der diachronen Kontrastierung von Englisch, Niederländisch, Isländisch, Schwedisch, Deutsch einschließlich Schweizerdeutsch sowie Luxemburgisch ist ein Spektrum an germanischen Sprachen abgedeckt, das alle Konstellationen erfasst: Isländisch hat gezeigt, dass zuviel phonologischer Umlaut eine morphologische Reanalyse behindert (neben dem $i$-Umlaut gab es auch $u$-Umlaut sowie verschiedene Brechungen). Auch gehen gleiche Umlautprodukte auf mehr als eine Ursache (z.B. den $i$-Umlaut) zurück, was die Ableitbarkeit des Umlauts erschwert. Der Umlaut wird im Isländischen seit vielen Jahrhunderten weitestgehend dort belassen, wo er lautgesetzlich entstanden war, was die Paradigmen stark irregularisiert hat. Schwedisch dagegen hat seine Umlautprodukte konsequenter beseitigt und nur in einigen häufig gebrauchten Paradigmen konserviert. Komplementär zum Isländischen hat das Schweizerdeutsche gezeigt, dass ,wohldosierter' phonologischer Umlaut (sog. Umlautfeindlichkeit) eher zu morphologischer Reanalyse einlädt (morphologische Umlautfreundlichkeit) als ein übergroßes Angebot womöglich verschiedener Umlautprozesse (Isländisch). Englisch und Niederländisch haben die Umlautprodukte beseitigt. Ursache ist ihre schwache phonologische Basis (im Niederländischen wurde nur kurzes $a$ und $u$ umlautet) sowie der phonologische Zusammenfall der Umlautprodukte mit anderen Phonemen (mangelnde Erkennbarkeit). Einen ganz anderen Weg jenseits von Konservierung und Eliminierung haben Deutsch und Luxemburgisch eingeschlagen: Hier wurde der Umlaut grammatikalisiert, d.h. mit festen Informationen befrachtet und in zahlreiche Paradigmen ausgedehnt, die ihn lautgesetzlich nicht produziert haben. Hier hat er auch autonomen Status erreicht insofern, als er, von Suffixen entkoppelt, allein die betreffenden Kategorien auszudrücken vermag (z.B. 'Plural', Typ Vater - Väter). Diese Pluralinformation via Umlaut hat sich im Deutschen soweit verselbstständigt, dass sie sogar in den Verbalbereich übergegangen ist (Transkategorialisierung, Typ muss - müssen). Während im Deutschen die Relation BasisvokalUmlautvokal noch relativ gut erkennbar ist, wurde sie im Luxemburgischen zerstört (arbitrarisiert), d.h. die Beziehung zwischen Basis- und Umlautvokal ist verwischt und verhält sich dem Ablaut vergleichbar. Dies hat die Funktionalisierung jedoch nicht beeinträchtigt. Sowohl im Deutschen als auch im Luxemburgischen ist der morphologische Umlaut noch (leicht) produktiv. 
Die germanischen (und romanischen) Sprachen eignen sich besonders gut für den Sprachwandelvergleich, da ihre Ausgangssprachen (Germanisch und mehr noch Latein) relativ gut bekannt sind. Umso erklärungsbedürftiger sind die einzelsprachlich divergierenden Entwicklungen. Während die Romanistik stark kontrastiv arbeitet, auch diachron, bestehen in der Germanistik noch viele Defizite.

\section{Zitierte Literatur}

Antonsen, Elmer (1969): „Zur Umlautfeindlichkeit des Oberdeutschen.“ In: Zeitschrift für Dialektologie und Linguistik 36, 201-207.

Augst, Gerhard (1971): „Über den Umlaut bei der Steigerung.“ In: Wirkendes Wort 21, 424-430.

Barðdal, Jóhanna, Nils Jörgensen, Gorm Larsen \& Bente Martinussen (1997): Nordiska. Våra språk förr och $n u$. Lund: Studentlitteratur.

Beito, Olav T. (1986): Nynorsk grammatikk. Lyd- og ordlare. Oslo: Det Norske Samlaget.

Braune, Wilhelm \& Ingo Reiffenstein $\left({ }^{15} 2004\right)$ : Althochdeutsche Grammatik I. Laut- und Formenlehre. Tübingen: Niemeyer (Sammlung kurzer Grammatiken germanischer Dialekte A.5/1).

Chapman, Carol (1994): „A diachronic argument against the split morphology hypothesis - analogical umlaut in German dialects." In: Transactions of the Philological Society, Bd. 92:1, 25-39.

Dal, Ingerid (1971): „Über den $i$-Umlaut im Deutschen.“ In: Ingerid Dal (Hg.): Untersuchungen zur germanischen und deutschen Sprachgeschichte, 31-45. Oslo: Universitetsforlaget.

Faiß, Klaus (1989): Englische Sprachgeschichte. Tübingen: Francke.

FAND = Fonologische Atlas van de Nederlandse Dialecten, hg. von Jan Goossens et al. Bd. I (1998), Bd. II und III (2000).

Fischer, Ludwig (1989): Luzerndeutsche Grammatik. Ein Wegweiser zur guten Mundart. Hitzkirch: Comenius.

Götz, Ursula (1996): „Zum analogen Umlaut im Bairischen.“ In: Sprachwissenschaft 21, 18-36.

Goossens, Jan (2000a): „Herauslösung und Herausbildung des Niederländischen.“ In: Heinz Eickmans, Loek Geeraedts \& Robert Peters (Hgg.): Ausgewählte Schriften zur niederländischen und deutschen Sprach- und Literaturwissenschaft, 197-211. München: Waxmann (Niederlande-Studien 22).

Goossens, Jan (2000b): „Konstituierendes in der Herausbildung der niederländischen Sprache.“ In: Heinz Eickmans, Loek Geeraedts \& Robert Peters (Hgg.): Ausgewählte Schriften zur niederländischen und deutschen Sprach- und Literaturwissenschaft, 213-228. München: Waxmann (Niederlande-Studien 22).

Goossens, Jan (2000c): „Umlaut en palatalisatie in den Nederlandse dialecten.“ In: Heinz Eickmans, Loek Geeraedts \& Robert Peters (Hgg.): Ausgewählte Schriften zur niederländischen und deutschen Sprach- und Literaturwissenschaft, 229-253. München: Waxmann (Niederlande-Studien 22).

Goossens, Jan (2000d): „Primaire en secundaire umlaut in het Nederlandse taalgebied.“ In: Heinz Eickmans, Loek Geeraedts \& Robert Peters (Hgg.): Ausgewählte Schriften zur niederländischen und deutschen Sprach- und Literaturwissenschaft, 255-259. München: Waxmann (Niederlande-Studien 22).

Haugen, Einar (1982): Scandinavian Language Structures. A Comparative Historical Survey. Tübingen: Niemeyer.

Holmes, Philip \& Hinchliffe, Jan (1998): Swedish. A Comprehensive Grammar. London/New York: Routledge.

Iversen, Ragnvald ( $\left.{ }^{7} 1990\right)$ : Norrøn grammatikk. Tano: Aschehoug. 
Iverson, Gregory \& Salmons, Joseph (2000): „Zur historischen Phonetik und Phonologie des Umlauts im Deutschen.“In: Zentrum für Allgemeine Sprachforschung Papers in Linguistics 15, 68-76.

Iverson, Gregory \& Salmons, Joseph (2009): „Naturalness and the Life Cycle of Language Change.“ In: Patrick Steinkrüger \& Manfred Krifka (Hgg.): On Inflection, 89-105. Berlin/New York: de Gruyter (Trends in Linguistics 184).

Jörg, Christine (1989): Isländische Konjugationstabellen. Hamburg: Buske.

Kastovsky, Dieter (1994): „Typological differences between English and German morphology and their causes.“ In: Toril Swan, Endre Mørck \& Olaf Jansen (Hgg.): Language Change and Language Structure: Older Germanic Languages in a Comparative Perspective, 135-157. Berlin/New York: de Gruyter (Trends in Linguistics 73).

Kazzazi, Kerstin (2004): „Welchen Weg die Füße nehmen. Überlegungen zum Umbau des Nominalsystems in den germanischen Sprachen.“ In: Maria Kozianka, Rosemarie Lühr \& Susanne Zeilfelder (Hgg.): Indogermanistik - Germanistik - Linguistik. Akten der Arbeitstagung der Indogermanischen Gesellschaft, Jena, 18. - 20.09.2002, 145-161. Hamburg: Kovač (Philologia 63).

Kunze, Konrad \& Damaris Nübling (Hgg.) (2009): Deutscher Familiennamenatlas, Band 1: Graphematik/Phonologie der Familiennamen I: Vokalismus. Berlin/New York: de Gruyter.

Lüssy, Heinrich (1974): Umlautprobleme im Schweizerdeutschen. Untersuchungen an der Gegenwartssprache. Frauenfeld: Huber.

Lüssy, Heinrich (1983): „Umlautung in den deutschen Dialekten.“ In: Werner Besch, Ulrich Knoop, Wolfgang Putschke \& Herbert Ernst Wiegand (Hgg.): Dialektologie. Ein Handbuch zur deutschen und allgemeinen Dialektforschung, 1083-1088. Berlin/New York: de Gruyter (Handbücher zur Sprach- und Kommunikationswissenschaft 1.2).

MAND = de Schutter, Georges, Boudewijn van den Berg, Ton Goeman \& Thera de Jong (Hgg.): Morfologische Atlas van de Nederlandse Dialecten. Bd. I (2005), Bd. II (2009).

Mottausch, Karl-Heinz (2002): „Umlaut und Entrundung im Altenglischen. Versuch einer Deutung.“ In: NOWELE 41, 3-16.

Moulton, William (1971): „Der morphologische Umlaut im Schweizerdeutschen.“ In: Maria Bindschedler (Hg.): Festschrift für Paul Zinsli, 15-25. Bern: Francke.

Nedoma, Robert (2006): Kleine Grammatik des Altisländischen. 2., erw. und aktualisierte Auflage. Heidelberg: Winter (Indogermanische Bibliothek 1).

Nübling, Damaris (2001): „Wechselflexion Luxemburgisch - Deutsch kontrastiv: ech soen - du sees/si seet vs. ich sage, du sagst, sie sagt. Zum sekundären Ausbau eines präsentischen Wurzelvokalwechsels im Luxemburgischen.“ In: Sprachwissenschaft 26, 433-472.

Nübling, Damaris (2005): „Forschungsperspektiven zur Nominalmorphologie deutscher Dialekte.“ In: Eckhard Eggers, Jürgen Erich Schmidt \& Dieter Stellmacher (Hgg.): Moderne Dialekte - Neue Dialektologie, 45-86. Stuttgart: Steiner (ZDL-Beiheft 130).

Nübling, Damaris (2006): „Zur Entstehung und Struktur ungebändigter Allomorphie: Pluralbildungsverfahren im Luxemburgischen.“ In: Claudine Moulin \& Damaris Nübling (Hgg.): Perspektiven einer linguistischen Luxemburgistik. Studien zu Synchronie und Diachronie, 107-128. Heidelberg: Winter (Germanistische Bibliothek 25).

Nübling, Damaris (2008): „Müssen, dürfen, können, mögen: Wie kam der Umlaut in die Präteritopräsentia? - Neues zu einem alten Problem der Irregularität.“ In: Cornelia Stroh \& Aina Urdze (Hgg.): Morphologische Irregularität. Neue Ansätze, Sichtweisen und Daten, 91-109. Bochum: Brockmeyer (Diversitas Linguarum 19).

Nübling, Damaris, Antje Dammel, Janet DukeRenata Szczepaniak ( $\left.{ }^{2} 2008\right)$ : Historische Sprachwissenschaft des Deutschen. Eine Einführung in die Prinzipien des Sprachwandels. 2. Auflage. Tübingen: Narr.

Paul, Hermann ( $\left.{ }^{25} 2007\right)$. Mittelhochdeutsche Grammatik. Neu bearbeitet von Thomas Klein, HansJoachim Solms \& Klaus-Peter Wegera. Mit einer Syntax von Ingeborg Schröbler, neubearbeitet und 
erweitert von Heinz-Peter Prell. Tübingen: Niemeyer (Sammlung kurzer Grammatiken germanischer Dialekte A.2).

Penzl, Herbert (1994): „Umlaut and secondary umlaut in Old High German.“ In: Language 25, 233-240. Pétursson, Magnús (1987): Lehrbuch der isländischen Sprache. Hamburg: Buske.

Ranke, Friedrich \& Dietrich Hofmann $\left({ }^{5} 1988\right)$ : Altnordisches Elementarbuch. Berlin: de Gruyter (Sammlung Göschen 2214).

Robinson, Orrin (1980): „An exception to Old High German umlaut.“ In: Kathryn Klar, Margaret Langdon \& Shirley Silver (Hgg.): American Indian and Indoeuropean Studies. Papers in Honor of Madison S. Beeler, 449-460. Den Hague: Mouton (Trends in Linguistics 16).

Ronneberger-Sibold, Elke (1990): „Zur Verselbständigung sprachlicher Einheiten: Der deutsche Umlaut.“ In: Norbert Boretzky, Werner Enninger \& Thomas Stolz (Hgg.): Spielarten der Natürlichkeit Spielarten der Ökonomie. Beiträge zum 5. Essener Kolloquium über „Grammatikalisierung: Natürlichkeit und Systemökonomie“,185-205. Bochum: Brockmeyer.

SAG = Teleman, Ulf, Staffan Hellberg, Erik Andersson \& Lisa Christensen (1999): Svenska Akademiens Grammatik, Del 2: Ord. Stockholm: Norstedts Ordbok.

Schirmunski, Victor (1962): Deutsche Mundartkunde. Vergleichende Laut- und Formenlehre der deutschen Mundarten. Berlin: Akademie.

Schnelzer, Klaus Otto (2008): Konjunktiv kontrastiv. Zur Morphologie bairischer und isländischer Verben. Regensburg: Edition Vulpes.

Schulte, Michael (1998): Grundfragen der Umlautphonemisierung. Eine strukturelle Analyse des nordgermanischen i/j-Umlauts unter Berücksichtigung der älteren Runeninschriften. Berlin/New York: de Gruyter.

Sonderegger, Stefan (1959): „Die Umlautfrage in den germanischen Sprachen.“ In: Kratylos 4, 1-12.

SDS III = Hotzenköcherle, Rudolf (Hg.) (1975): Sprachatlas der deutschen Schweiz, Band III: Formengeographie. Bern: Francke.

Steffens, Rudolf (2010): „Beobachtungen zum Umlaut in der frühneuhochdeutschen Mainzer Stadtsprache." In: Claudine Moulin, Fausto Ravida \& Nikolaus Ruge (Hgg.): Sprache in der Stadt. Akten der 25. Tagung des Internationalen Arbeitskreises Historische Stadtsprachenforschung. Luxemburg, 11.-13. Oktober 2007, 297-329. Heidelberg: Winter (Germanistische Bibliothek 36).

Szczepaniak, Renata (2007): Der phonologisch-typologische Wandel des Deutschen von einer Silben- zu einer Wortsprache. Berlin/New York: de Gruyter (Studia Linguistica Germanica 85).

Weber, Albert (1987): Zürichdeutsche Grammatik. Zürich: Schweizer Spiegel.

Wessén, Elias (1992): Svensk språkhistoria. Edsbruk: Akademitryck.

Wurzel, Wolfgang Ullrich (1984): „Was bezeichnet der Umlaut im Deutschen?“ In: Zeitschrift für Phonetik, Sprachwissenschaft und Kommunikationsforschung 37, 647-663. 